hep-th/0303207

\title{
Chiral Rings and Phases of Supersymmetric Gauge Theories
}

\author{
Freddy Cachazo, Nathan Seiberg and Edward Witten \\ School of Natural Sciences, Institute for Advanced Study, Princeton NJ 08540 USA
}

\begin{abstract}
We solve for the expectation values of chiral operators in supersymmetric $U(N)$ gauge theories with matter in the adjoint, fundamental and anti-fundamental representations. A simple geometric picture emerges involving a description by a meromorphic one-form on a Riemann surface. The equations of motion are equivalent to a condition on the integrality of periods of this form. The solution indicates that all semiclassical phases with the same number of $U(1)$ factors are continuously connected.
\end{abstract}

March 2003 


\section{Introduction}

In the previous century, it became clear that supersymmetric field theories exhibit rich dynamics which is amenable to exact analysis (for a review, see e.g. [1]). A renaissance of this subject has recently been stimulated by the work of Dijkgraaf and Vafa [2], who, motivated by earlier developments [3-9], conjectured an interesting relation between SUSY gauge theories and matrix models. Many authors have added to this framework matter in the fundamental representation [10-32]. Here we will continue the investigation in our previous papers [33,27, 34], in which these theories have been studied by focusing on the chiral ring and the relations which follow from the anomaly. As we will see, these relations can be explicitly solved.

We will study a supersymmetric $U(N)$ gauge theory with chiral superfields consisting of a multiplet $\Phi$ in the adjoint representation, plus $N_{f}$ copies of the fundamental representation (quarks, denoted $Q^{f}, f=1, \ldots, N_{f}$ ) and the anti-fundamental representation (anti-quarks, denoted $\widetilde{Q}_{f}$ ). We take the superpotential to be

$$
W_{\text {tree }}=\operatorname{Tr} W(\Phi)+\widetilde{Q}_{\widetilde{f}} m_{f}^{\widetilde{f}}(\Phi) Q^{f},
$$

where we suppressed the color indices in the second term, and $W(\Phi)$ and $m_{f}^{\widetilde{f}}(\Phi)$ are polynomials. Classically the theory has several vacua. First, the fundamental and antifundamental fields $Q$ and $\widetilde{Q}$ can vanish while $\Phi$ is a diagonal matrix whose eigenvalues are at the stationary points of $W(\Phi)$. In these vacua, the quarks and anti-quarks are massive, and the microscopic $U(N)$ gauge symmetry is broken to $\prod_{i} U\left(N_{i}\right)$ with $\sum_{i} N_{i}=N$. The second kind of classical vacua involve nonzero expectation values of $Q$ and $\widetilde{Q}$. Here the gauge symmetry is broken to $\prod_{i} U\left(N_{i}\right)$ with $\sum_{i} N_{i}<N$. In the quantum theory the unbroken $\prod_{i} S U\left(N_{i}\right)$ confines and the low energy spectrum includes a number of $U(1)$ multiplets.

Our main tool in analyzing the dynamics is the chiral ring of the theory. Interesting bosonic operators in this ring are the gauge invariant observables [33,27]

$$
\begin{aligned}
T(z) & =\operatorname{Tr} \frac{1}{z-\Phi} \\
R(z) & =-\frac{1}{32 \pi^{2}} \operatorname{Tr} \frac{W_{\alpha} W^{\alpha}}{z-\Phi} \\
M(z)_{\widetilde{f}}^{f} & =\widetilde{Q}_{\widetilde{f}} \frac{1}{z-\Phi} Q^{f} .
\end{aligned}
$$


Classically, $z$ takes values in the complex plane, and these observables have simple poles where $z$ equals an eigenvalue of $\Phi$. In the quantum theory, some of the poles become cuts and although an a priori reason is not really understood, it turns out that $z$ can be analytically continued through the cuts to a second sheet. The two-sheeted plane is a Riemann surface $\Sigma$ which is described by the equation

$$
y^{2}=W^{\prime}(z)^{2}+f(z)
$$

where $R(z)$ of (1.2) is given by $\left(W^{\prime}(z)-y(z)\right) / 2$. In (1.3), $W^{\prime}(z)$ and $f(z)$ are polynomials of degree $n$ and $n-1$ respectively, and (1.3) describes a genus $n-1$ Riemann surface.

The exact solution corresponds to solving for the polynomial $f(z)$, which depends only on the vacuum of the theory. However, it is often useful to consider the "off-shell" theory for arbitrary values of the $n$ coefficients of this polynomial. Alternatively, we can parametrize the solution by the $n$ periods

$$
S_{i}=\frac{1}{2 \pi i} \oint_{A_{i}} R(z) d z,
$$

where $A_{i}$ are $A$-cycles of $\Sigma$. Semiclassically, these $A$-cycles originate from the poles of the classical theory, which quantum mechanically became cuts in the $z$ plane. Important objects in our discussion are the integers

$$
N_{i}=\frac{1}{2 \pi i} \oint_{A_{i}} T(z) d z,
$$

which are the ranks of the various unbroken gauge groups.

In the following sections we describe the theory in more detail and solve off-shell, using relations in the chiral ring, for $T(z)$ and $M(z)$, which were defined in (1.2). One of the surprises we encounter is that $T(z)$ has a very simple analytic structure: its only singularities are simple poles with integer residues. This is particularly surprising for the poles in the second sheet which are not even visible classically. We then view $T(z) d z$ as a one-form on $\Sigma$ and explore its periods. Integrality of the periods in (1.5) plus "modular invariance" (invariance under exchanges of the periods that arise when parameters in the superpotential are varied) suggests that all periods of $T(z) d z$ might be integers. In particular, the other compact periods of $T$

$$
b_{i}=-\frac{1}{2 \pi i} \oint_{B_{i}} T(z) d z,
$$


which were introduced and interpreted physically in [34], are integers on-shell. The constraints on the analytic structure of $T(z) d z$ and its periods allow us to find an explicit expression for it.

We then compute the off-shell effective superpotential as a function of $S_{i}$ using the matrix model. It is satisfying to find out that the equations of motion of $S_{i}$ which are derived from this superpotential coincide with the previously imposed geometric condition about the periods of $T(z) d z$. This result is consistent with the fact that (1.6) is satisfied only on-shell and that imposing (1.6) allows us to completely solve for our observables.

In [34], we explored the phases of the theory with $\Phi$ only (and no quarks) as the parameters in the tree level superpotential are varied. Some of the phases of the theory were distinguished by the behavior of Wilson and 't Hooft loops which probe the different kinds of confinement. Here, since the theory includes fundamental matter fields, there is no real confinement, and we might expect that there are no phase transitions [35,36]. We will use the term "pseudo-confining" to describe a phase in which $Q$ and $\widetilde{Q}$ have no classical expectation values and the individual low energy $S U\left(N_{i}\right)$ theories are confining, though the microscopic $U(N)$ theory is not. The result of [35, 36] is the possibility of smooth interpolation between Higgs and pseudo-confinement.

This subject has also been discussed in $\mathcal{N}=2$ supersymmetric theories with matter, first with $S U(2)$ gauge theories [37], and later with other gauge groups [38]. It was shown that the massless particles at special points in the moduli space can be interpreted either as elementary quarks or as magnetic monopoles. More explicitly, by varying the bare mass of the quarks, one can continuously interpolate between a limit in which it is more natural to interpret the massless particles as electrically charged to a limit in which it is more natural to interpret them as magnetic. When $\mathcal{N}=2$ is broken to $\mathcal{N}=1$ by a mass term for the adjoint fields, these quarks/monopoles condense. In one limit, where the condensed particles are electrically charged, it is natural to interpret the condensation as a Higgs mechanism. In the other limit, where the condensed objects are interpreted as magnetic monopoles, the condensation looks in the low energy pure $S U(2)$ theory as confinement, but in the full theory including the massive quarks, there is no precise order parameter for confinement, and we will call this limit pseudo-confining. Therefore, by changing the bare mass of the quarks, one can continuously interpolate between Higgs and pseudo-confinement.

The situation we have just described is a special case of a more general story we will describe below. We will show that as we vary the parameters in $m(\Phi)$, we can connect 
almost all the classical limits of the theory with the same number of $U(1)$ factors in the low energy spectrum.

The poles of $T(z)$ play a crucial role in the interpolation from one classical limit to another. A pole in the first sheet represents a classical nonzero expectation value of $Q$, i.e., a Higgs phenomenon. A pole in the second sheet corresponds to an expectation value of $Q$ that arises only from quantum corrections. In the quantum theory, the Riemann surface is smooth and there is no physical boundary between the first and the second sheet. The poles can continuously move from one sheet to another, a phenomenon which represents the continuous interpolation between the Higgs mechanism and pseudo-confinement.

By analogy with [34], we explore the various classical limits of each branch by performing modular transformations on $\Sigma$. Unlike the situation in [34], because of the presence of the fundamental matter, $T$ has poles and $\Sigma$ should be thought of as a Riemann surface with punctures. This makes the relevant modular group bigger. Using appropriate modular transformations, we can connect all values of $b_{i}$ with fixed $N_{i}$; we can also connect most values of $N_{i}$. Therefore, the quantum theory has many fewer phases than the classical theory.

\section{Preliminaries}

We consider an $\mathcal{N}=1$ supersymmetric $U(N)$ gauge theory with chiral superfields consisting of an adjoint multiplet $\Phi, N_{f}$ fundamentals $Q^{f}$, and $N_{f}$ anti-fundamentals $\widetilde{Q}_{\widetilde{f}}$ ( $f$ and $\tilde{f}$ are the flavor indices). As in the introduction, the tree level superpotential is

$$
W_{\text {tree }}=\operatorname{Tr} W(\Phi)+\widetilde{Q}_{\widetilde{f}_{f}} m_{f}^{\tilde{f}}(\Phi) Q^{f}
$$

The function $W$ and the matrix $m$ are taken to be polynomials

$$
\begin{aligned}
W(z) & =\sum_{k=0}^{n} \frac{1}{k+1} g_{k} z^{k+1} \\
m_{f}^{\widetilde{f}}(z) & =\sum_{k=1}^{l+1} m_{f, k}^{\widetilde{f}} z^{k-1}
\end{aligned}
$$

It is convenient to define the polynomial

$$
B(z)=\operatorname{det} m(z)
$$


We denote its degree by $L$ and its roots by $z_{I}(I=1, \ldots, L)$. We will see from the solution of the theory that some of its observables depend only on $B(z)$ and not on the details of the matrix $m_{f} \widetilde{f}(z)$. L will play the role of an effective number of flavors. We assume that $m_{f}^{\widetilde{f}}(z)$ is sufficiently generic so that $B(z)$ does not have double roots, and none of its roots coincide with those of $W^{\prime}(z)$.

Some special cases are the following:

1. No flavors. This is the basic example which is most widely studied. When $W(\Phi)=0$, this theory has $\mathcal{N}=2$ supersymmetry.

2. No $\Phi$ or equivalently $W(\Phi)=M \Phi^{2}$ with large $M$. This theory is $\mathcal{N}=1$ SQCD.

3. $m_{f}^{\widetilde{f}}(z)=\left(\sqrt{2} z+m_{f}\right) \delta_{f}^{\tilde{f}}$. Here the theory without $W(\Phi)$ has $\mathcal{N}=2$ supersymmetry.

4. $m_{f}^{\widetilde{f}}(z)=z^{l} \delta_{f}^{\widetilde{f}}$ with $W(\Phi)=0$. This theory was studied in [39].

Let us examine the vacua of the theory in the semiclassical approximation. We parametrize the superpotential of the adjoint field as

$$
W^{\prime}(z)=g_{n} \prod_{i=1}^{n}\left(z-a_{i}\right)
$$

in terms of its stationary points $a_{i}$. A first set of vacua, which we will refer to loosely as "pseudo-confining vacua," arise from

$$
\begin{aligned}
& \langle Q\rangle=\langle\widetilde{Q}\rangle=0
\end{aligned}
$$

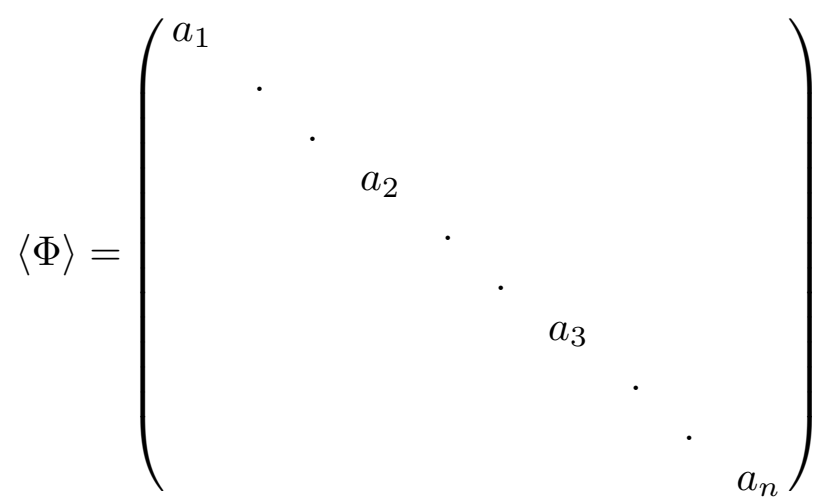

where $a_{i}$ occurs $N_{i}$ times $\left(\sum_{i} N_{i}=N\right)$. Here, the fundamental $U(N)$ gauge group is broken to $\prod_{i} U\left(N_{i}\right)$. At low energies the $S U\left(N_{i}\right)$ confine, leading to $\prod_{i} N_{i}$ vacua in which the low energy gauge group is $U(1)^{k}$. $k$ can be less than $n$ if some of the $a_{i}$ do not appear in (2.5) and the corresponding $N_{i}$ are equal to zero.

There are also Higgs vacua in which $Q$ and $\widetilde{Q}$ have a vacuum expectation value at the classical level, and one of the diagonal elements of $\langle\Phi\rangle$ is equal to a zero of $B(z)$, say $z_{1}$. 
In explaining how this works, in order not to clutter the equations, we consider the simple case of $N_{f}=1$. The choice

$$
\begin{aligned}
& \langle Q\rangle=\left(\begin{array}{llllll}
h & 0 & . & \cdot & . & 0
\end{array}\right) \\
& \langle\widetilde{Q}\rangle=\left(\begin{array}{llllll}
\widetilde{h} & 0 & \cdot & . & . & 0
\end{array}\right) \\
& \langle\Phi\rangle=\left(\begin{array}{ccccccccc}
z_{1} & & & & & & & & \\
& a_{1} & & & & & & & \\
& & \cdot & & & & & & \\
& & a_{2} & & & & & \\
& & & \cdot & & & & \\
& & & & \cdot & & & \\
& & & & & a_{3} & & \\
& & & & & & \cdot & \\
& & & & & & \cdot & \\
& & & & & & & a_{n}
\end{array}\right)
\end{aligned}
$$

gives a classical vacuum when

$$
\widetilde{h} h=-\frac{W^{\prime}\left(z_{1}\right)}{B^{\prime}\left(z_{1}\right)} .
$$

In such a vacuum, the fundamental $U(N)$ gauge group is broken to $\prod_{i} U\left(N_{i}\right)$ with $\sum_{i} N_{i}=$ $N-1$.

We cannot have two different eigenvalues of $\langle\Phi\rangle$, say $\left\langle\Phi^{1}{ }_{1}\right\rangle$ and $\left\langle\Phi^{2}{ }_{2}\right\rangle$, equal to $z_{1}$. In this case $\left\langle Q_{1,2}\right\rangle$ and $\left\langle\widetilde{Q}^{1,2}\right\rangle$ must be nonzero, but then the equation of motion of $\Phi^{2}{ }_{1}$ is not satisfied.

More generally, for each zero $z_{I}$ of $B(z)$, we let $r_{I}$ denote the number of eigenvalues of $\Phi$ that are equal to $z_{I}$. In a classical vacuum, each $r_{I}$ can be either zero or one. Clearly,

$$
0 \leq \sum_{I=1}^{L} r_{I} \leq L
$$

and

$$
N=\sum_{I=1}^{L} r_{I}+\sum_{i=1}^{n} N_{i}
$$

We will refer to vacua with nonzero $r_{I}$ as "Higgs vacua." Again, at low energies $\prod_{i} S U\left(N_{i}\right)$ confine and lead to $\prod_{i} N_{i}$ vacua with gauge group $U(1)^{k}$. We will see below that in the quantum theory the pseudo-confining vacua (2.5) are continuously connected to the Higgs vacua (2.6). 
We will be interested in the chiral operators [33,27]

$$
\begin{aligned}
T(z) & =\operatorname{Tr} \frac{1}{z-\Phi} \\
w_{\alpha}(z) & =\frac{1}{4 \pi} \operatorname{Tr} \frac{W_{\alpha}}{z-\Phi} \\
R(z) & =-\frac{1}{32 \pi^{2}} \operatorname{Tr} \frac{W_{\alpha} W^{\alpha}}{z-\Phi} \\
M(z)_{\widetilde{f}}^{f} & =\widetilde{Q}_{\widetilde{f}} \frac{1}{z-\Phi} Q^{f}
\end{aligned}
$$

These composite operators are defined initially in terms of a power series in $\frac{1}{z}$ around infinity, but turn out to have an interesting analytic continuation. All these operators are $\mathcal{O}(1 / z)$ for $z \rightarrow \infty$.

First, let us examine these observables in the classical theory. For $T$, we easily find

$$
T_{c l}(z)=\sum_{i} \frac{N_{i}}{z-a_{i}}+\sum_{I} \frac{r_{I}}{z-z_{I}} .
$$

We will view $T(z) d z$ as a one-form. Classically, its only singularities are simple poles at $a_{i}, z_{I}$ and infinity with residues $N_{i}, r_{I}$ and $-N$, respectively. Generalizing (2.7), we find

$$
M_{c l}(z)=-\sum_{I=1}^{L} \frac{r_{I} W^{\prime}\left(z_{I}\right)}{z-z_{I}} \frac{1}{2 \pi i} \oint_{z_{I}} \frac{1}{m(x)} d x
$$

i.e. there are poles only at $z_{I}$ with $r_{I}=1$. In contrast to poles of $T$, the poles of $M_{c l}$ have residues that depend on the details of the interactions. The classical limit of $R(z)$ is zero, since it is proportional to a fermion bilinear.

Now let us give a preview of what will occur in the quantum theory. Quantum mechanically, when the fermion bilinear in the numerator of $R(z)$ gets an expectation value, one might expect $R(z)$ to develop a singularity at $a_{i}$ and/or (in a Higgs vacuum) $z_{I}$ because the denominator of $R(z)$ is singular when $z$ approaches an eigenvalue of $\Phi$. We will see that actually $R(z)$ has no singularities at $z_{I}$, while the singularities of $R(z)$ associated with $a_{i}$ are replaced by cuts that shrink to $a_{i}$ in a weak coupling limit; we let $A_{i}$ denote a cycle that circles counterclockwise once around the $i^{t h}$ such cut. Because of the cuts, $R(z)$ is naturally defined on a Riemann surface $\Sigma$ that is a double cover of the $z$-plane. Any value of $z$, such as $z=z_{I}$, really corresponds to a pair of points on $\Sigma$; we denote the points with $z=z_{I}$ as $q_{I}$, which is on the first sheet and visible semiclassically, and $\widetilde{q}_{I}$, which is on the second sheet and invisible semiclassically. 
$M$ and $T$ are also naturally defined on this double cover, and as functions on $\Sigma$, their only singularities turn out to be simple poles. The classical pole of $T$ at $a_{i}$ with residue $N_{i}$ is replaced by a quantum statement $\frac{1}{2 \pi i} \oint_{A_{i}} T(z) d z=N_{i}$; in effect, these poles disappear when the points $a_{i}$ are replaced by cuts and the $z$-plane by its double cover $\Sigma$. The other classical singularities of $T$ are the poles at those $z_{I}$ with $r_{I}=1$; these are also the locations of the only classical singularities of $M$. What happens to these singularities quantum mechanically? They remain as simple poles and, roughly speaking, they do not move from their classical locations at $z_{I}$. To be more precise, these poles appear quantum mechanically at the points $q_{I}$ that lie above $z_{I}$ on the first sheet of $\Sigma$. The residues of $T(z) d z$ at these poles are equal to 1 quantum mechanically just as classically; this actually follows by an argument similar to one in [33]. Indeed, letting $C_{I}$ denote a small contour around $q_{I}$, the contour integral

$$
\frac{1}{2 \pi i} \oint_{C_{I}} T(z) d z=\frac{1}{2 \pi i} \oint_{C_{I}} \operatorname{Tr} \frac{1}{z-\Phi} d z
$$

is really a $c$-number, not subject to quantum fluctuations, since it is equal to the number of eigenvalues of $\Phi$ enclosed by the contour. If the only singularity of $T(z)$ enclosed by $C_{I}$ is a simple pole, the residue must hence be one.

On the other hand, the residue of the pole of $M$ does receive quantum corrections; it is not protected by any such argument, because of the quark operators in the numerator of $M$. In addition to the singularities at $q_{I}, T$ and $M$ will turn out in general to have poles, crucial in the consistency of the whole picture, at the points $\widetilde{q}_{I}$ on the second sheet. The meaning of these poles is somewhat mysterious from a semi-classical point of view, as the second sheet is invisible classically. Quantum mechanically, they reflect the fact that the Higgs phases can be reached from the pseudo-confining phase by analytic continuation.

\section{Anomaly Equations}

To verify and extend these statements, we will use the relations obeyed by the operators (2.10) in the chiral ring. These operators satisfy the anomaly equations [34,27]

$$
\begin{aligned}
& {\left[W^{\prime}(z) T(z)\right]_{-}+\operatorname{tr}\left[m^{\prime}(z) M(z)\right]_{-}=2 R(z) T(z)+w_{\alpha}(z) w^{\alpha}(z)} \\
& {\left[W^{\prime}(z) w_{\alpha}(z)\right]_{-}=2 R(z) w_{\alpha}(z)} \\
& {\left[W^{\prime}(z) R(z)\right]_{-}=R(z)^{2}} \\
& {\left[(M(z) m(z))_{f}^{f^{\prime}}\right]_{-}=R(z) \delta_{f}^{f^{\prime}}} \\
& {\left[(m(z) M(z))_{\widetilde{f}}^{\widetilde{f}^{\prime}}\right]_{-}=R(z) \delta_{\widetilde{f}}^{\widetilde{f^{\prime}}}}
\end{aligned}
$$


Here $m(z)$ and $M(z)$ are matrices in flavor space. We multiply such matrices as $(A B)_{f^{\prime \prime}}^{f}=$ $A_{f^{\prime}}^{f} B_{f^{\prime \prime}}^{f^{\prime}}$ and $\operatorname{tr}$ denotes a trace over the flavor indices. Below we will solve these equations. We start by solving the third equation in (2.14). We write it as

$$
W^{\prime}(z) R(z)+\frac{1}{4} f(z)=R(z)^{2}
$$

with a polynomial $f(z)=-4\left[W^{\prime}(z) R(z)\right]_{+}$of degree $n-1$. Its solution is

$$
2 R(z)=W^{\prime}(z)-\sqrt{W^{\prime}(z)^{2}+f(z)} .
$$

This solution is parameterized by the $n$ coefficients in $f(z)$. We see that $R(z)$ has cuts in the complex $z$ plane. In the semiclassical approximation of small $f$, each cut $A_{i}$ is naturally associated with a zero of $W^{\prime}, a_{i}$.

It is natural to analytically continue $z$ through the cuts to a second sheet. The doublesheeted complex plane is a Riemann surface $\Sigma$. Define

$$
y(z)=W^{\prime}(z)-2 R(z)
$$

and write (2.16) as an equation for a Riemann surface $\Sigma$

$$
y^{2}=W^{\prime}(z)^{2}+f(z)
$$

This genus $n-1$ Riemann surface was introduced in [6]. We can naturally understand $\Sigma$ as a double cover of the complex $z$-plane branched at the roots of $W^{\prime}(z)^{2}+f(z)$. There are $n$ branch cuts; as above we denote as $A_{i}$ a contour that circles around the $i^{\text {th }}$ cut. Most of the functions we will consider in this work have singularities at infinity; if the points on $\Sigma$ with $z=\infty$ are removed, the $n$ cycles $A_{i}$ with $i=1, \ldots, n$ are independent. Instead of parameterizing $\Sigma$ by the coefficients in $f$, we alternatively parameterize it by the variables

$$
S_{i}=\frac{1}{2 \pi i} \oint_{A_{i}} R(z) d z \quad i=1, \ldots, n .
$$

Each point on the complex $z$-plane labeled by a given value of $z$ corresponds to a pair of points on $\Sigma$. To specify a point $q$ on $\Sigma$, we must give its $z$ coordinate $z(q)$ and also its $y$ coordinate $y(q)$. For each point $q$ on the first sheet, there is an image point $\widetilde{q}$ on the second sheet, with $z(\widetilde{q})=z(q)$ and $y(\widetilde{q})=-y(q)$. Actually, the terminology "first sheet" and "second sheet" is useful primarily in a semiclassical limit in which $z$ is large; 
more generally we would simply refer to a pair of points $q$ and $\widetilde{q}$ with the same value of $z$. Clearly, we have also

$$
\begin{aligned}
& W^{\prime}(q)=W^{\prime}(\widetilde{q}) \\
& R(q)+R(\widetilde{q})=W^{\prime}(z) .
\end{aligned}
$$

We will sometimes adopt a convenient though slightly imprecise notation, writing $z=q$ when we mean $z=z(q)$ and $y=y(q)$, or $z \rightarrow q$ when we mean $z \rightarrow z(q)$ and $y \rightarrow y(q)$.

The branch of the square root in (2.16) is chosen such that for large $z$ in the first sheet we recover the semiclassical answer

$$
R(z) \approx-\frac{f(z)}{4 W^{\prime}(z)}=\frac{S}{z}+\mathcal{O}\left(1 / z^{2}\right)
$$

(A simple contour deformation argument shows that $S=\sum_{i} S_{i}$.) Then, using (2.20) it is easy to see that the asymptotic limit in the second sheet is

$$
R(z)=W^{\prime}(z)+\mathcal{O}(1 / z)=g_{n} z^{n}+\mathcal{O}\left(z^{n-1}\right) .
$$

\section{Solving For $M(z)$ And $T(z)$}

In this section, we consider $R(z)$ of (2.16) as given and solve for $M(z)$. This means that we solve for $M(z)$ as a function of $S_{i}$. Moreover, in this section, we will find a solution which corresponds in the semiclassical limit to the pseudo-confining vacua (2.5); in other words, we set all $r_{I}=0$. For such solutions, we impose that $M(z)$ and therefore also $T(z)$ are regular in the first sheet except for the cuts. As we will see, other solutions correspond to the Higgs vacua (2.6). We will discuss below the question of whether these branches are connected to the pseudo-confining vacua.

\section{Solving For $M(z)$}

$M(z)$ is solved from the last two equations in (2.14)

$$
\begin{aligned}
& {\left[(M(z) m(z))_{f}^{f^{\prime}}\right]_{-}=R(z) \delta_{f}^{f^{\prime}}} \\
& {\left[(m(z) M(z))_{\widetilde{f}}^{\tilde{f}}\right]_{-}=R(z) \delta_{\widetilde{f}}^{\widetilde{f}^{\prime}} .}
\end{aligned}
$$

We determine the polynomials $[m M]_{+}$and $[M m]_{+}$such that $M(z)$ is regular in the first sheet [27]. 
We claim that the solution with these boundary conditions is

$$
M(z)=-\sum_{i=1}^{n} \frac{1}{2 \pi i} \oint_{A_{i}} \frac{R(x)}{x-z} \frac{1}{m(x)} d x
$$

where $A_{i}$ are the cuts in $R(z)$. To prove it, we note that the integrand in (3.2) decays as $\mathcal{O}\left(1 / x^{2}\right)$ at infinity in the first sheet (with the branch of the square root given in (2.16)) and therefore by contour deformation, (3.2) is equivalent to

$$
M(z)=-\sum_{i=1}^{n} \frac{1}{2 \pi i} \oint_{A_{i}} \frac{R(x)}{x-z} \frac{1}{m(x)} d x=R(z) \frac{1}{m(z)}-\sum_{I=1}^{L} \frac{R\left(q_{I}\right)}{z-z_{I}} \frac{1}{2 \pi i} \oint_{z_{I}} \frac{1}{m(x)} d x .
$$

To obtain this formula, we have used contour deformation on the first sheet; the term $R(z) / m(z)$ comes from the pole of the function $1 /(x-z)$, and the second term comes from the poles of matrix elements of $1 / m(x)$. These latter poles are at $z=z_{I}$, the points at which det $m=0$. Each point $z=z_{I}$ corresponds to a pair of points $q_{I}, \widetilde{q}_{I}$ on $\Sigma$, where $q_{I}$ is on the first sheet and $\widetilde{q}_{I}$ on the second; since the contour deformation in (3.3) has been done on the first sheet, the residue at $z=z_{I}$ involves $R\left(q_{I}\right)$, the value of $R$ on the first sheet. To verify that (3.3) satisfies (3.1), we proceed as follows. Multiplying the right hand side of (3.3) by $m(z)$ from the right or the left, the first term contributes $R(z)$ times a unit matrix. And the second term leads to a polynomial in $z$ because

$$
\begin{aligned}
\frac{m(z)}{z-z_{I}} \oint_{z_{I}} \frac{1}{m(x)} d x & =\frac{m(z)-m\left(z_{I}\right)}{z-z_{I}} \oint_{z_{I}} \frac{1}{m(x)} d x+\frac{m\left(z_{I}\right)}{z-z_{I}} \oint_{z_{I}} \frac{1}{m(x)} d x \\
& =\frac{m(z)-m\left(z_{I}\right)}{z-z_{I}} \oint_{z_{I}} \frac{1}{m(x)} d x+\frac{1}{z-z_{I}} \oint_{z_{I}} m(x) \frac{1}{m(x)} d x \\
& =\frac{m(z)-m\left(z_{I}\right)}{z-z_{I}} \oint_{z_{I}} \frac{1}{m(x)} d x
\end{aligned}
$$

is a polynomial in $z$. This verifies that our formula for $M(z)$ does obey (3.1).

Because of the polynomial ambiguity in (3.1), its solution is not unique. The particular solution (3.2) of was chosen to be regular at the points $q_{I}$, which lie at large values $z=z_{I}$ that (if we are near a semiclassical limit) are outside the integration contours in (3.2). Regularity at $q_{I}$ is, as we have seen, the right behavior for the pseudo-confining solutions with $r_{I}=0$. However, the solution (3.2) actually has a pole at the points $\widetilde{q}_{I}$ on the second sheet. This behavior is evident in (3.3): as $R\left(\widetilde{q}_{I}\right) \neq R\left(q_{I}\right)$, the singularities of the two terms on the right hand side of $(3.3)$ do not cancel at $\widetilde{q}_{I}$, though they cancel at $q_{I}$. At first sight, the meaning of the singularity of $M(z)$ on the second sheet is rather mysterious, 
since the second sheet is invisible classically. Its interpretation will ultimately become clear.

\section{Solving For $T(z)$}

Having found $R(z)$ and $M(z)$, we can solve for $T(z)$. We solve for $T(z)$ as a function of $S_{i}$. The polynomial ambiguity in the equations is determined by imposing that $\frac{1}{2 \pi i} \oint_{A_{i}} T(z) d z=N_{i}$. Since we use the solution for $M(z)$ given by (3.2) or equivalently by (3.3), the result obtained here is relevant to the pseudo-confining vacua with $r_{I}=0$.

First we need to calculate $\left[\operatorname{tr} m^{\prime}(z) M(z)\right]_{-}$with $M(z)$ given in (3.3). We get

$$
\begin{aligned}
{[\operatorname{tr} \quad} & \left.m^{\prime}(z) M(z)\right]_{-}=\left[R(z) \operatorname{tr} m^{\prime}(z) \frac{1}{m(z)}\right]_{-}-\sum_{I=1}^{L}\left[\frac{R\left(q_{I}\right)}{z-z_{I}} \frac{1}{2 \pi i} \oint_{z_{I}} \operatorname{tr} m^{\prime}(z) \frac{1}{m(x)} d x\right]_{-} \\
& =R(z) \frac{B^{\prime}(z)}{B(z)}-\sum_{I=1}^{L} \frac{R\left(q_{I}\right)}{z-z_{I}}-\sum_{I=1}^{L}\left[R\left(q_{I}\right) \operatorname{tr} \frac{m^{\prime}(z)-m^{\prime}\left(z_{I}\right)}{z-z_{I}} \frac{1}{2 \pi i} \oint_{z_{I}} \frac{1}{m(x)} d x\right]_{-} \\
& =\frac{B^{\prime}(z)\left(W^{\prime}(z)-y(z)\right)}{2 B(z)}-\sum_{I=1}^{L} \frac{W^{\prime}\left(z_{I}\right)-y\left(q_{I}\right)}{2\left(z-z_{I}\right)} .
\end{aligned}
$$

We used the relation $\operatorname{tr} m^{\prime} \frac{1}{m}=\frac{B^{\prime}}{B}$ and the fact that the first two terms in the second line have only negative powers of $z$ and the third term is polynomial in $z$.

Since $B$ and $W^{\prime}$ are polynomials and the zeros of $B$ are $z_{I}$,

$$
\frac{B^{\prime}(z) W^{\prime}(z)}{2 B(z)}-\sum_{I=1}^{L} \frac{W^{\prime}\left(z_{I}\right)}{2\left(z-z_{I}\right)}=\left[\frac{B^{\prime}(z) W^{\prime}(z)}{2 B(z)}\right]_{+}=\left[\frac{B^{\prime}(z) y(z)}{2 B(z)}\right]_{+},
$$

where the last step is correct only in the first sheet (on which $W^{\prime}-y$ vanishes for large $z)$. Therefore (3.5) becomes

$$
\left[\operatorname{tr} m^{\prime}(z) M(z)\right]_{-}=-\left[\frac{B^{\prime}(z) y(z)}{2 B(z)}\right]_{-}+\sum_{I=1}^{L} \frac{y\left(q_{I}\right)}{2\left(z-z_{I}\right)} .
$$

Supersymmetric vacua are described by solutions of (2.14) with $w_{\alpha}(z)=0$. So the first equation in (2.14) reduces

$$
\left[W^{\prime}(z) T(z)\right]_{-}-2 R(z) T(z)+\operatorname{tr} \quad\left[m^{\prime}(z) M(z)\right]_{-}=0
$$

and can be written as

$$
[y(z) T(z)]_{-}=-\operatorname{tr}\left[m^{\prime}(z) M(z)\right]_{-}=\left[\frac{B^{\prime}(z) y(z)}{2 B(z)}\right]_{-}-\sum_{I=1}^{L} \frac{y\left(q_{I}\right)}{2\left(z-z_{I}\right)} .
$$


Hence

$$
T(z)=\frac{B^{\prime}(z)}{2 B(z)}-\sum_{I=1}^{L} \frac{y\left(q_{I}\right)}{2 y(z)\left(z-z_{I}\right)}+\frac{c(z)}{y(z)}
$$

where

$$
c(z)=\left\langle\operatorname{Tr} \frac{W^{\prime}(z)-W^{\prime}(\Phi)}{z-\Phi}\right\rangle-\frac{1}{2} \sum_{I=1}^{L} \frac{W^{\prime}(z)-W^{\prime}\left(z_{I}\right)}{z-z_{I}}
$$

is a polynomial of degree $n-1$.

For large $z$, the first term in (3.11) behaves as $N g_{n} z^{n-1}$ since $\langle\operatorname{Tr} \mathbf{1}\rangle=N$. The second term behaves as $-\frac{1}{2} L g_{n} z^{n-1}$. This is enough to determine the large $z$ behavior of $T(z)$,

$$
T(z)= \begin{cases}\frac{N}{z}+\mathcal{O}\left(\frac{1}{z^{2}}\right) & \text { in the first sheet } \\ \frac{L-N}{z}+\mathcal{O}\left(\frac{1}{z^{2}}\right) & \text { in the second sheet } .\end{cases}
$$

For the pseudo-confining vacua, we expect $T(z)$ to be regular at the points $q_{I}$ on the first sheet. This indeed follows from (3.10), as $y\left(q_{I}\right) / y(z) \rightarrow 1$ for $z \rightarrow q_{I}$. On the second sheet, since $y\left(q_{I}\right) / y(z) \rightarrow-1$ for $z \rightarrow \widetilde{q}_{I}, T(z)$ has a pole, $T(z) \sim 1 /\left(z-z_{I}\right)$ for $z \rightarrow q_{I}$. Why the residue of this pole is precisely 1 will be clear when we compare to the Higgs vacua.

Finally, $c(z)$ can be determined by the requirements

$$
\frac{1}{2 \pi i} \oint_{A_{i}} T(z) d z=N_{i}
$$

Let $P$ denote the point $z=\infty$ on the first sheet and $\widetilde{P}$ the point $z=\infty$ on the second sheet. We can summarize the singularities of $T$ as follows: $T(z) d z$ has simple poles at $P$, $\widetilde{P}$, and $\widetilde{q}_{I}$ with residues $-N,-L+N$ and 1 respectively. Elsewhere it is regular.

\section{Higgs Vacua And Singularities In The First Sheet}

Consider varying the parameters in $m_{f}^{\widetilde{f}}(z)$ with fixed $W(\Phi)$ and fixed $S_{i}$. The Riemann surface $\Sigma$ is unchanged but the zeros $z_{I}$ of $B\left(z_{I}\right)$ change. Let us move one of them, say $z_{1}$, through one of the cuts, say $A_{1}$. Of the two points $q_{1}$ and $\widetilde{q}_{1}$ on $\Sigma$ that correspond to $z=z_{1}$, our solutions for $M$ and $T$ have poles at $\widetilde{q}_{1}$, which is on the second sheet, but not at $q_{1}$. When $z_{1}$ passes through the cut, the two points $q_{1}$ and $\widetilde{q}_{1}$ are exchanged. We end up with solutions for $M$ and $T$ which obey the equations but do not satisfy the boundary conditions that we have imposed so far; they are singular at $q_{1}$.

This process has a simple physical interpretation. We started semiclassically with $\langle\Phi\rangle$ whose eigenvalues, as shown in (2.5), are approximately equal to the roots $a_{i}$ of $W^{\prime}$; this 
corresponds to having all $r_{I}=0$. In a semiclassical limit, the cut $A_{1}$ is near $a_{1}$. When $z_{1}$ passes through the cut, it is near $a_{1}$ and the solution (2.5) is near the solution (2.6) that describes the Higgs branch. At this stage, the strong quantum dynamics are important and a semiclassical treatment is not precise enough. Passing $z_{1}$ through the cut and taking it to be again large (or at least far away from all cuts), we may find ourselves in a Higgs branch with $r_{1}=1$. On this branch, $M$ and $T$ are expected to have poles on the first sheet. Thus, our proposal is that in a process in which $z_{1}$ moves through one of the cuts, a branch with $r_{1}=0$ is continuously transformed into a branch with $r_{1}=1$.

Let us see how this occurs in terms of our solution. The analytic continuation of (3.3) is completely clear: when $z_{1}$ moves through the cuts, $q_{1}$ and $\widetilde{q}_{1}$ are exchanged. Thus, (3.3) transforms into

$$
M(z)=R(z) \frac{1}{m(z)}-\frac{R\left(\widetilde{q}_{1}\right)}{z-z_{1}} \frac{1}{2 \pi i} \oint_{z_{1}} \frac{1}{m(x)} d x-\sum_{I=2}^{L} \frac{R\left(q_{I}\right)}{z-z_{I}} \frac{1}{2 \pi i} \oint_{z_{I}} \frac{1}{m(x)} d x .
$$

To derive this from (3.2), we note that as $z_{1}$ moves, the cut $A_{1}$ must be continuously deformed to avoid $q_{1}$ and $\widetilde{q}_{1}$. By the time $q_{1}$ and $\widetilde{q}_{1}$ have been exchanged, $A_{1}$ has transformed to a contour $A_{1}^{\prime}=A_{1}+C_{1}-\widetilde{C}_{1}$, where $C_{1}$ and $\widetilde{C}_{1}$ are small contours that, respectively, encircle $q_{1}$ and $\widetilde{q}_{1}$ in the counterclockwise directions. (3.2) is thus replaced by

$$
M(z)=-\sum_{i=1}^{n} \frac{1}{2 \pi i} \oint_{A_{i}} \frac{R(x)}{x-z} \frac{1}{m(x)} d x+\frac{1}{2 \pi i} \oint_{C_{1}} \frac{R(x)}{x-z} \frac{1}{m(x)} d x+\frac{1}{2 \pi i} \oint_{\widetilde{C}_{1}} \frac{R(x)}{x-z} \frac{1}{m(x)} d x .
$$

The extra two terms in (4.2) have the effect of cancelling the singularity at $\widetilde{q}_{1}$ and adding a singularity at $q_{1}$.

Similarly, the singularity of $T(z)$ in the second sheet at $\widetilde{q}_{1}$ moves to the first sheet to $q_{1}$. Thus, after $z_{1}$ passes through the $A_{1}$ cut, we have $\frac{1}{2 \pi i} \oint_{\widetilde{C}_{1}} T(z) d z=0, \frac{1}{2 \pi i} \oint_{C_{1}} T(z) d z=1$. In the course of the transition, the integers $N_{i}=\frac{1}{2 \pi i} \oint_{A_{i}} T(z) d z$ cannot change. After the transition, $A_{1}$ has transformed to $A_{1}^{\prime}=A_{1}+C_{1}-\widetilde{C}_{1}$, so after the transition $N_{1}=$ $\frac{1}{2 \pi i} \oint_{A_{1}^{\prime}} T(z) d z$ and hence $N_{1}-1=\frac{1}{2 \pi i} \oint_{A_{1}} T(z) d z$. Thus, the effective value of $N_{1}$ has been reduced by 1 in this transition.

More generally, for arbitrary $r_{I}=0,1$, the solution for $M$ becomes

$$
\begin{aligned}
M(z) & =R(z) \frac{1}{m(z)}-\sum_{I=1}^{L}\left(1-r_{I}\right) \frac{R\left(q_{I}\right)}{z-z_{I}} \frac{1}{2 \pi i} \oint_{z_{I}} \frac{1}{m(x)} d x-\sum_{I=1}^{L} r_{I} \frac{R\left(\widetilde{q}_{I}\right)}{z-z_{I}} \frac{1}{2 \pi i} \oint_{z_{I}} \frac{1}{m(x)} d x \\
& =R(z) \frac{1}{m(z)}-\sum_{I=1}^{L} \frac{r_{I} W^{\prime}\left(z_{I}\right)+\left(1-2 r_{I}\right) R\left(q_{I}\right)}{z-z_{I}} \frac{1}{2 \pi i} \oint_{z_{I}} \frac{1}{m(x)} d x
\end{aligned}
$$



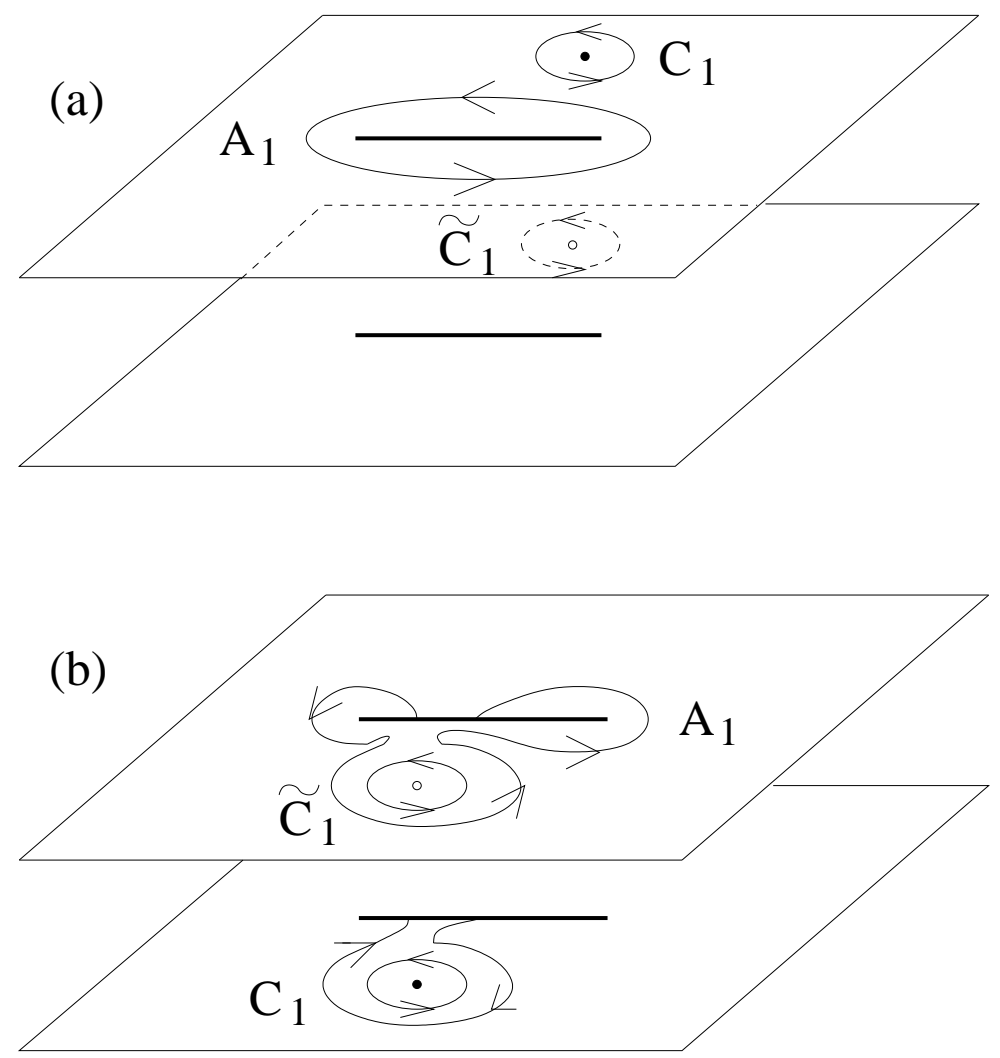

Fig. 1: (a) Original configuration with $q_{1}$ on the upper sheet (black dot) enclosed by the $C_{1}$ contour and $\widetilde{q}_{1}$ on the lower sheet (white dot) enclosed by the $\widetilde{C}_{1}$ contour. Also shown is the first cut (wide black line) enclosed by the $A_{1}$ contour. (b) Same configuration as in $(a)$ after $q_{1}$ and $\widetilde{q}_{1}$ are passed through the first cut. The $A_{1}$ contour is deformed as shown. A new $A_{1}^{\prime}$ cycle that only encloses the cut is then given by $A_{1}^{\prime}=A_{1}+C_{1}-\widetilde{C}_{1}$

where we used $(2.20)$ to express $R\left(\widetilde{q}_{I}\right)=W^{\prime}\left(z_{I}\right)-R\left(q_{I}\right)$. We would like to make a few comments about this expression:

1. $M(z)$ has a singularity with residue $r_{I}\left(2 R\left(q_{I}\right)-W^{\prime}\left(z_{I}\right)\right) \frac{1}{2 \pi i} \oint_{z_{I}} \frac{1}{m(x)} d x$ at $q_{I}$ and a singularity with residue $\left(r_{I}-1\right)\left(2 R\left(q_{I}\right)-W^{\prime}\left(z_{I}\right)\right) \frac{1}{2 \pi i} \oint_{z_{I}} \frac{1}{m(x)} d x$ at $\widetilde{q}_{I}$. In particular, singularities are at $\widetilde{q}_{I}$ if $r_{I}=0$ and at $q_{I}$ if $r_{I}=1$.

2. In the classical limit of zero $R(z)$, this expression coincides with our classical answer (2.12). Note that the residue of $M$ at $q_{I}$ is $r_{I}\left(2 R\left(q_{I}\right)-W^{\prime}\left(z_{I}\right)\right) \frac{1}{2 \pi i} \oint_{z_{I}} \frac{1}{m(x)} d x$, which is corrected from the classical answer $-r_{I} W^{\prime}\left(z_{I}\right) \frac{1}{2 \pi i} \oint_{z_{I}} \frac{1}{m(x)} d x$.

3. It is easy to check, as in (3.4), that the terms which are summed over $I$ in (4.3) have the property that when they are multiplied by $m(z)$ they lead to a polynomial in $z$. These terms change the poles in $M$ and modify the polynomials $[m M]_{+}$and $[M m]_{+}$, but do not affect the anomaly equations (3.1). 
By repeating the procedure that was used to find (3.10), or by simply analytically continuing (3.10) to let the $z_{I}$ pass through the cuts, we find that the general solution for $T$ is

$$
T(z)=\frac{B^{\prime}(z)}{2 B(z)}-\sum_{I=1}^{L} \frac{\left(1-2 r_{I}\right) y\left(q_{I}\right)}{2 y(z)\left(z-z_{I}\right)}+\frac{c(z)}{y(z)} .
$$

We have used the fact that when $z_{I}$ passes through a cut and $r_{I}$ becomes $1, y\left(q_{I}\right)$ is transformed to $-y\left(q_{I}\right)=\left(1-2 r_{I}\right) y\left(q_{I}\right)$. As expected, for $r_{I}=0$ the function is regular at $q_{I}$ and has a pole with residue one at $\widetilde{q}_{I}$, while for $r_{I}=1$ it is regular at $\widetilde{q}_{I}$ and has a pole with residue one at $q_{I}$. The number of singularities in the first sheet is $\sum_{I} r_{I}$ and satisfies $L \geq \sum_{I} r_{I} \geq 0$ in agreement with the classical reasoning in (2.8)(2.9).

By interpreting the analytic continuation of the pseudo-confining vacua as $z_{I}$ pass through cuts as physical Higgs vacua, we can shed light on many otherwise mysterious features of the solutions found in section 3 for the pseudo-confining vacua. For example, the singularities on the second sheet now have a rationale; upon analytic continuation, they become semiclassical singularities on the first sheet in Higgs vacua. It is also now clear why the residues of poles of $T(z) d z$ on the second sheet are precisely 1: this is needed to agree with the semiclassical behavior in Higgs vacua.

All of our analysis has been carried out at fixed $S_{i}$. This corresponds to treating the field theory off-shell; an on-shell analysis, by contrast, would involve extremizing the superpotential as a function of the $S_{i}$. In effect, in our analysis, we have ignored the fact that the $S_{i}$ will change when the $z_{I}$ are varied. In this off-shell analysis, we have found that $N_{i}$ can be reduced, for any given $i$, by passing the $z_{I}$ through the $i^{\text {th }}$ cut $A_{i}$. Clearly, there must be a limit to this process, for if it is performed too many times, $N_{i}$ will become negative. Intuitively, we expect that if the $N_{i}$ are large enough then the back-reaction on the $S_{i}$ in moving a single $z_{I}$ through a cut is small. How far can one expect to go before the back-reaction becomes significant? We conjecture but will not try to prove in this paper that a given $N_{i}$ can be reduced all the way to 1 by passing $N_{i}-1$ of the $z_{I}$ through the cut $A_{i}$. One cannot expect to go farther, since if $N_{i}$ changes from 1 to 0 , this would change the number of $U(1)$ 's in the low energy gauge group, so such a process cannot occur continuously. It must be that if one tries to transform $N_{i}$ all the way to zero, the cut $A_{i}$ will close up at the last step. We return briefly to this issue in section 8 . 


\section{5. $T(z) d z$ As A Differential And The Complete Solution}

By definition, $T(z) d z$ is a meromorphic differential on the Riemann surface $\Sigma$ of genus $n-1$ that was given in (2.18):

$$
y^{2}=W^{\prime}(z)^{2}+f(z)
$$

$T(z) d z$ has simple poles at $P, \widetilde{P}$ and $\widetilde{q}_{I}$ with residues $-N, N-L$ and 1 respectively. We are now going to introduce some formalism for describing $T$ in more detail. This will eventually be used in section 7 to prove that on-shell, the periods of $T$ are all integers. At the end of the present section, we will assume this statement and deduce some consequences.

Ignoring the punctures of $\Sigma$ at $P$ and $\widetilde{P}$, a complete basis of independent $A$-cycles of $\Sigma$ are the $A_{i}$ for $i=1, \ldots, n-1$. If $p_{1}$ and $p_{2}$ are any chosed points in $\Sigma$, then the RiemannRoch or index theorem can be used to show that meromorphic differentials on $\Sigma$ with poles at $p_{1}$ and $p_{2}$ and no other singularities do exist. By normalizing such a differential, we can make the residues at $p_{1}$ and $p_{2}$ precisely -1 and 1 ; by adding a holomorphic differential, we can in a unique fashion make the $A_{i}$ periods vanish. Hence, there exists a unique meromorphic differential $\tau_{p_{1}, p_{2}}$ whose only singularities on $\Sigma$ are simple poles at $p_{1}$ and $p_{2}$ with residues -1 and 1 respectively and $\oint_{A_{i}} \tau_{p_{1}, p_{2}}=0$.

Since $T(z) d z$ is singular only at $\widetilde{q}_{I}, P$ and $\widetilde{P}$, the differential

$$
T(z) d z-\left(N \tau_{P, \widetilde{P}}+\sum_{I=1}^{L} \tau_{\widetilde{P}, \widetilde{q}_{I}}\right)
$$

is a holomorphic one-form on $\Sigma$. Using a basis for holomorphic differentials $\left\{\zeta_{1}, \ldots \zeta_{n-1}\right\}$ on $\Sigma$ which is characterized by $\oint_{A_{i}} \zeta_{k}=\delta_{i k}$, we have

$$
T(z) d z=N \tau_{P, \widetilde{P}}+\sum_{I=1}^{L} \tau_{\widetilde{P}, \widetilde{q}_{I}}+\sum_{j=1}^{n-1} h_{j} \zeta_{j}
$$

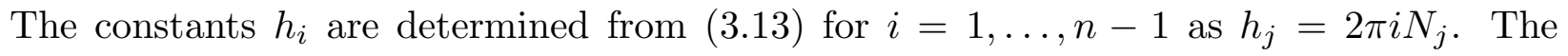
condition on $A_{n}$ is automatically satisfied. To see this note that on the first sheet the integral around $A_{n}$ can be deformed to an integral around $P$ and $-A_{1}-\ldots-A_{n-1}$. This leads to $\frac{1}{2 \pi i} \oint_{A_{n}} T(z) d z=N-N_{1}-\ldots-N_{n-1}=N_{n}$. We conclude that

$$
T(z) d z=N \tau_{P, \widetilde{P}}+\sum_{I=1}^{L} \tau_{\widetilde{P}, \widetilde{q}_{I}}+2 \pi i \sum_{j=1}^{n-1} N_{j} \zeta_{j} .
$$

We now consider the noncompact cycles $\left\{\widehat{B}_{1}, \ldots, \widehat{B}_{n}\right\}$ with the intersection pairing $A_{i} \cap \widehat{B}_{j}=\delta_{i j}, \widehat{B}_{i} \cap \widehat{B}_{j}=0$ (see figure 2). It is convenient to use the basis $\left\{B_{1}, \ldots, B_{n-1}, \widehat{B}_{n}\right\}$ defined by $B_{i}=\widehat{B}_{i}-\widehat{B}_{n}$. Clearly, $B_{i}$ are compact cycles and together with $A_{i}$ for $i=1, \ldots, n-1$ form a canonical basis of $H_{1}(\Sigma)$. 


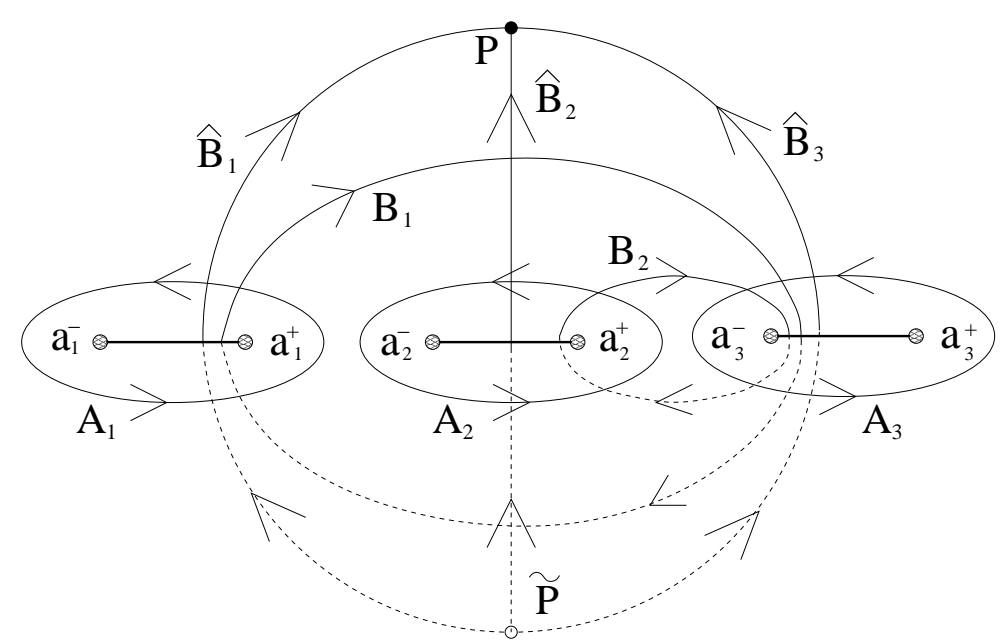

Fig. 2: Riemann Surface $\Sigma$ for $n=3$ as a double cover of the $z$ complex plane. Solid lines are on the first sheet while dashed lines are on the second sheet. Gray circles represent the location of the branching points of (5.1). The $i^{\text {th }}$ branch cut runs between the points $a_{i}^{-}$and $a_{i}^{+}$which collapse to the $i^{t h}$ root, $a_{i}$, of $W^{\prime}(z)$ when $f(z)$ is taken to zero. $\left\{A_{1}, A_{2}, A_{3}, \widehat{B}_{1}, \widehat{B}_{2}, \widehat{B}_{3}\right\}$ form a basis of one cycles, on the surface with punctures, with canonical intersection pairing. $\left\{A_{1}, A_{2}, B_{1}, B_{2}, \widehat{B}_{3}\right\}$ is a different choice of basis. $\left\{A_{1}, A_{2}, B_{1}, B_{2}\right\}$ is the canonical basis of one cycles for a compact Riemann surface of genus 2 . The points $P$ and $\widetilde{P}$ with coordinates $z(P)=z(\widetilde{P})=\infty$ and $y(P)=-y(\widetilde{P})$ are depicted as a black dot and a white dot respectively.

Consider first the period

$$
\oint_{B_{k}} T(z) d z=N \oint_{B_{k}} \tau_{P, \widetilde{P}}+\sum_{I=1}^{L} \oint_{B_{k}} \tau \widetilde{P}, \widetilde{q}_{I}+2 \pi i \sum_{j=1}^{n-1} N_{j} \oint_{B_{k}} \zeta_{j}
$$

Using the Riemann bilinear relations, we have that for any $\tau_{p_{1}, p_{2}}$ (see appendix B for a review),

$$
\frac{1}{2 \pi i} \oint_{B_{k}} \tau_{p_{1}, p_{2}}=\int_{p_{2}}^{p_{1}} \zeta_{k}
$$

where the contour in the right hand side is as follows. If $p_{1}$ and $p_{2}$ are on the same sheet, the contour is on that sheet. If $p_{1}$ and $p_{2}$ are on different sheets the contour crosses through cut number $n$. In both cases winding around the $A_{k}$ cycles can lead to an integer ambiguity. This ambiguity corresponds to the fact that in order to define the differentials $\tau_{p_{1}, p_{2}}$ and to derive the Riemann bilinear relations a particular choice of representative of one cycles has been made. A different choice leads to an answer which differs by an integer. See appendix A for a more detailed discussion of this issue. 
Using (5.6) in the first and second terms of (5.5) and the fact that $\oint_{B_{k}} \zeta_{j}=\oint_{B_{j}} \zeta_{k}$ in the last term of (5.5) we get,

$$
\frac{1}{2 \pi i} \oint_{B_{k}} T(z) d z=N \int_{\widetilde{P}}^{P} \zeta_{k}+\sum_{I=1}^{L} \int_{\widetilde{q_{I}}}^{\widetilde{P}} \zeta_{k}+\sum_{j=1}^{n-1} N_{j} \oint_{B_{j}} \zeta_{k} .
$$

Since the first and the last term of (5.7) can be combined

$$
N \int_{\widetilde{P}}^{P} \zeta_{k}+\sum_{j=1}^{n-1} N_{j} \oint_{B_{j}} \zeta_{k}=N \int_{\widehat{B}_{n}} \zeta_{k}+\sum_{j=1}^{n-1} N_{j} \oint_{B_{j}} \zeta_{k}=\sum_{j=1}^{n} N_{j} \int_{\widehat{B}_{j}} \zeta_{k},
$$

(5.7) can be written as

$$
\frac{1}{2 \pi i} \oint_{B_{k}} T(z) d z=\sum_{j=1}^{n} N_{j} \int_{\widehat{B}_{j}} \zeta_{k}+\sum_{I=1}^{L} \int_{\widetilde{q}_{I}}^{\widetilde{P}} \zeta_{k} .
$$

Finally, we can compute the period of $T(z) d z$ on the non-compact cycle $\widehat{B}_{n}$. This period is infinite and requires a regularization. We will introduce regularized contours $\widehat{B}_{i}^{r}$, as contours from $\widetilde{\Lambda}_{0}$ to $\Lambda_{0}$ passing through the $i^{\text {th }}$ cut. $\Lambda_{0}$ is a complex number with sufficiently large $\left|\Lambda_{0}\right| . \quad \Lambda_{0}$ and $\widetilde{\Lambda}_{0}$ are the points in the first and the second sheet which correspond to the same value of $\Lambda_{0}$. Only the behavior up to $\mathcal{O}\left(1 / \Lambda_{0}\right)$ will be relevant for us in the computations.

Using (5.4), we have

$$
\int_{\widehat{B}_{n}^{r}} T(z) d z=\int_{\widehat{B}_{n}^{r}}\left(N \tau_{P, \widetilde{P}}+\sum_{I=1}^{L} \tau_{\widetilde{P}, \widetilde{q}_{I}}+2 \pi i \sum_{i=1}^{n-1} N_{i} \zeta_{i}\right) .
$$

We want to write it as an integral of $\tau_{P, \widetilde{P}}$ over various cycles. In the last term we use $\int_{\widehat{B}_{n}^{r}} \zeta_{i}=\int_{\widetilde{\Lambda}_{0}}^{\Lambda_{0}} \zeta_{i}=\frac{1}{2 \pi i} \oint_{B_{i}} \tau_{\Lambda_{0}, \widetilde{\Lambda}_{0}}$. In the second term we use the Riemann bilinear relations

$$
\int_{S}^{R} \tau_{T Q}=\int_{Q}^{T} \tau_{R S}
$$

(recall that here and in the following expressions with line integrals there is an ambiguity in $2 \pi i$ times an integer which is discussed in appendix A) to write

$$
\int_{\widetilde{\Lambda}_{0}}^{\Lambda_{0}} \tau_{\widetilde{P}, \widetilde{q}_{I}}=\int_{\widetilde{q}_{I}}^{\widetilde{P}} \tau_{\Lambda_{0}, \widetilde{\Lambda}_{0}}=\int_{\widetilde{q}_{I}}^{\widetilde{P}} \tau_{P, \widetilde{\Lambda}_{0}}+\mathcal{O}\left(1 / \Lambda_{0}\right)
$$


Finally, we note that

$$
\int_{\widetilde{q}_{I}}^{\widetilde{P}} \tau_{P, \widetilde{\Lambda}_{0}}=\int_{\widetilde{q}_{I}}^{\widetilde{\Lambda}_{0}} \tau_{P, \widetilde{P}}+\pi i+\mathcal{O}\left(1 / \Lambda_{0}\right)
$$

See appendix A for a proof of this result.

Combining these results, 5.10 can be written as,

$$
\int_{\widehat{B}_{n}^{r}} T(z) d z=N \int_{\widetilde{\Lambda}_{0}}^{\Lambda_{0}} \tau_{P, \widetilde{P}}+\pi i L+\sum_{i=1}^{n-1} N_{i} \oint_{B_{i}} \tau_{P, \widetilde{P}}+\sum_{I=1}^{L} \int_{\widetilde{q}_{I}}^{\widetilde{\Lambda}_{0}} \tau_{P, \widetilde{P}}+\mathcal{O}\left(1 / \Lambda_{0}\right) .
$$

where we have used that $\oint_{B_{i}} \tau_{\Lambda_{0}, \widetilde{\Lambda}_{0}}=\oint_{B_{i}} \tau_{P, \widetilde{P}}+\mathcal{O}\left(1 / \Lambda_{0}\right)$.

This can be brought to the same form as (5.9) by using that $B_{i}=\widehat{B}_{i}^{r}-\widehat{B}_{n}^{r}$,

$$
\int_{\widehat{B}_{n}^{r}} T(z) d z=\sum_{j=1}^{n} N_{j} \int_{\widehat{B}_{j}^{r}} \tau_{P, \widetilde{P}}+\sum_{I=1}^{L} \int_{\widetilde{q}_{I}}^{\widetilde{\Lambda}_{0}} \tau_{P, \widetilde{P}}+\pi i L+\mathcal{O}\left(1 / \Lambda_{0}\right) .
$$

Equations (5.9) and (5.15) for the periods of $T(z) d z$ will be shown to be related to the field equations coming from the field theory effective superpotential in section 7 . The field equations imply that $\frac{1}{2 \pi i} \oint_{B_{i}} T(z) d z$ are integers. Here we will assume this fact and show that it is enough to determine all the remaining parameters.

Assuming that $\frac{1}{2 \pi i} \oint_{B_{i}} T(z) d z$ is an integer for all $i=1, \ldots, n-1$ and recalling (3.13), it follows that $T(z) d z$ has integer periods over all compact cycles of $\Sigma$. Therefore $T(z) d z$ has to be a logarithmic derivative of a meromorphic function on $\Sigma: T(z) d z=d \log \psi(z)$ for some $\psi(z) . \psi$ should have a pole at $P$ of order $N$ and zeros at $\widetilde{P}$ and $\widetilde{q}_{I}$ of order $N-L$ and 1 respectively. For $L \leq 2 N$ such a function can be constructed as follows [19]

$$
\psi(z)=P(z)+\sqrt{P^{2}(z)-\alpha B(z)},
$$

where the degree $N$ polynomial $P(z)$ and the constant $\alpha$ must be determined.

The condition that (5.16) be single valued on $\Sigma$ is

$$
\begin{aligned}
& P^{2}(z)-\alpha B(z)=F(z) H^{2}(z) \\
& W^{\prime}(z)^{2}+f(z)=F(z) Q^{2}(z),
\end{aligned}
$$

where $Q(z), H(z)$ and $F(z)$ are polynomials in $z$. Indeed, these conditions imply that $\sqrt{P^{2}-\alpha B}=y H(z) / Q(z)$.

The degree of $Q(z)$ corresponds to the number of $N_{i}$ 's that are taken to be zero. When a given $N_{i}$ is zero, the corresponding branch cut in $y(z)$ closes up. 
Special cases are the following.

1. $\operatorname{deg} Q(z)=0$ : This corresponds to the case with all $N_{i} \neq 0$ and the $\operatorname{deg} W^{\prime}(z) \leq N$.

2. $\operatorname{deg} H(z)=0$ : This corresponds to the case when the group is completely broken to $U(1)^{N}$ and $\operatorname{deg} W^{\prime}(z) \geq N$.

Using that

$$
T(z) d z=\partial_{z} \chi d z ; \quad \psi(z)=e^{\chi(z)} .
$$

we can explicitly compute the period of $T(z) d z$ on $\widehat{B}_{n}^{r}$,

$$
\int_{\widehat{B}_{n}^{r}} T(z) d z=\log \left(\frac{4}{\alpha B_{L}} \Lambda_{0}^{2 N-L}\right)+\mathcal{O}\left(\frac{1}{\Lambda_{0}}\right)
$$

where $B_{L}$ is defined such that $B(z)=B_{L} \prod\left(z-z_{I}\right)$. This result is consistent with the asymptotic behavior of $T(z)$ given in (3.12).

In order to determine $\alpha$, note that $B_{L}$ has mass dimension $N_{f}-L$, so $\alpha$ must have dimension $2 N-N_{f}$. The only parameter with mass dimension that does not depend on the details of the superpotential is the scale $\Lambda$ of the theory. So $\alpha$ is equal to $\Lambda^{2 N-N_{f}}$ up to a numerical constant. This constant depends on the subtraction scheme. We choose a scheme where the standard $\mathcal{N}=2$ result for the curve $(\underline{5.17})$ is recovered when $m(z)_{f}^{\widetilde{f}}=$

$\sqrt{2}\left(z+m_{f}\right) \delta_{f}^{\tilde{f}}$. Note that here $m_{f}$ is not the canonically normalized mass. This choice leads to $\alpha / 4=2^{-N_{f} / 2} \Lambda^{2 N-N_{f}}$. It is convenient to define $\widehat{B}(z)=2^{-N_{f} / 2} B(z)$ such that the final result for $T(z)$ is given by,

$$
\left\langle\operatorname{Tr} \frac{1}{z-\Phi}\right\rangle=\frac{\partial}{\partial z} \log \left(P(z)+\sqrt{P^{2}(z)-4 \Lambda^{2 N-N_{f}} \widehat{B}(z)}\right) .
$$

Through equation (5.17) we can now determine also $f(z)$ and therefore $y(z)$ and $R(z)$. This means that all the observables of the theory are now determined. Of course, we still need to justify the claim that the compact periods of $T$ are all integers. We postpone this to section 7 .

\section{Matrix Model Computations}

In this section, we will explicitly compute the effective superpotential from the matrix model. 
Following [27], only the sphere and disk contribution to the free energy of the corresponding matrix model are relevant. Let us denote them $\mathcal{F}_{0}$ and $\mathcal{F}_{1}$ respectively. Explicitly, we have to compute

$\exp \left(-\frac{\widehat{N}^{2}}{S^{2}} \mathcal{F}_{0}-\frac{\widehat{N}}{S} \mathcal{F}_{1}+\ldots\right)=\frac{1}{\operatorname{vol} U(\widehat{N})} \int \frac{d \widehat{\Phi}}{\Lambda^{N^{2}}} \frac{d \widehat{Q} d \widehat{\widetilde{Q}}}{\nu^{\widehat{N} N_{f}}} \exp \left(-\frac{\widehat{N}}{S}[\operatorname{Tr} W(\widehat{\Phi})+\widehat{\widetilde{Q}} m(\widehat{\Phi}) \widehat{Q}]\right)$

where $\Lambda$ is a dimension one constant which will later be identified with the dynamically generated scale of the field theory. $\nu$ is defined such that if all quarks are taken to have masses equal to $\Lambda$, i.e. $m(\widehat{\Phi})_{f}^{\widetilde{f}}=\Lambda \delta_{f}^{\widetilde{f}}$, then the matrix integral over them is equal to one; i.e.

$$
\nu=\int d x d \bar{x} e^{-\widehat{N} \Lambda x \bar{x} / S}=\frac{\pi S}{\widehat{N} \Lambda}=\exp \left(-\log \frac{\Lambda \widehat{N}}{\pi S}\right)
$$

where $x$ and $\bar{x}$ are two integration variables 1 .

For large $\widehat{N}$ the volume of $U(\widehat{N})$ is 40,41 ,

$$
\operatorname{vol} U(\widehat{N})=\exp \left(-\frac{\widehat{N}^{2}}{2}\left[\log \frac{\widehat{N}}{2 \pi}-\frac{3}{2}\right]+\mathcal{O}(1)\right)
$$

Therefore,

$$
\frac{1}{\Lambda^{N^{2}} \operatorname{vol} U(\widehat{N})}=\exp \left(-\frac{\widehat{N}^{2}}{S^{2}}\left[\frac{1}{2} S^{2}\left(\log \frac{2 \pi \Lambda^{2}}{\widehat{N}}+\frac{3}{2}\right)\right]+\mathcal{O}(1)\right)
$$

At weak coupling the potential in (6.1) has several minima. We will focus on an expansion around minima where $\widehat{N}_{i}$ of the eigenvalues of $\widehat{\Phi}$ are at $a_{i}$ and $\widehat{Q}=\widehat{\widetilde{Q}}=0$. There are also minima with $r_{I}=0,1$ eigenvalues of $\widehat{\Phi}$ at $z_{I}$ and nonzero $\widehat{Q}, \widehat{\widetilde{Q}}$.

The integral over $\widehat{Q}$ and $\widehat{\widetilde{Q}}$ can be explicitly carried out for diagonal $\widehat{\Phi}$ with eigenvalues $\lambda_{k}$

$$
\int d \widehat{Q} d \widehat{\widetilde{Q}} \exp \left(-\frac{\widehat{N}}{S} \widehat{\widetilde{Q}} m(\widehat{\Phi}) \widehat{Q}\right)=\exp \left(-\frac{\widehat{N}}{S}\left[N_{f} S \log \frac{\widehat{N}}{\pi S}+\frac{S}{\widehat{N}} \sum_{k=1}^{\widehat{N}} \log B\left(\lambda_{k}\right)\right]\right) .
$$

1 We can also redefine $\widehat{Q}$ and $\widetilde{Q}$ such that

$$
\exp \left(-\frac{\widehat{N}^{2}}{S^{2}} \mathcal{F}_{0}-\frac{\widehat{N}}{S} \mathcal{F}_{1}+\ldots\right)=\frac{1}{\operatorname{vol} U(\widehat{N})} \int \frac{d \widehat{\Phi}}{\Lambda^{\widehat{N}^{2}}} \frac{d \widehat{Q} d \widehat{\widetilde{Q}}}{\nu^{\widehat{N} N_{f}}} \exp \left(-\frac{\widehat{N}}{S} \operatorname{Tr} W(\widehat{\Phi})-\widehat{\widetilde{Q}} m(\widehat{\Phi}) \widehat{Q}\right)
$$

and $\nu=\pi / \Lambda$. 
Recall that $B(z)$ has been defined in (2.3) to be equal to the determinant of $m(z)$.

Reducing the integration over $\widehat{\Phi}$ to its eigenvalues produces the Vandermonde determinant. This implies that the only integration left in (6.1) can be written as

$$
\lim _{\widehat{N} \rightarrow \infty} \int \prod_{k=1}^{\widehat{N}} d \lambda_{k} \exp \left(-\frac{\widehat{N}}{S} \sum_{k=1}^{\widehat{N}} W\left(\lambda_{k}\right)+2 \sum_{i<j} \log \left(\lambda_{i}-\lambda_{j}\right)-\sum_{k=1}^{\widehat{N}} \log B\left(\lambda_{k}\right)\right) .
$$

This integral can be evaluated using a saddle point approximation and taking into account the measure factors gives

$\exp \left(-\frac{\widehat{N}^{2}}{S^{2}}\left[\int d \lambda \rho(\lambda) W(\lambda)-\iint d \lambda d \lambda^{\prime} \rho(\lambda) \rho\left(\lambda^{\prime}\right) \log \left|\frac{\lambda-\lambda^{\prime}}{\Lambda}\right|\right]-\frac{\widehat{N}}{S}\left[\int d \lambda \rho(\lambda) \log \frac{B(\lambda)}{\Lambda^{N_{f}}}\right]\right)$

where $\rho(\lambda)$ is the eigenvalue distribution normalized to $\int \rho(\lambda) d \lambda=S$. This density function gives $\widehat{N}_{i}$ eigenvalues in the $i^{\text {th }}$ cut.

From $(6.8), \mathcal{F}_{0}$ and $\mathcal{F}_{1}$ can be identified. For $\mathcal{F}_{0}$ we have

$$
\mathcal{F}_{0}=\int d \lambda \rho(\lambda) W(\lambda)-\iint d \lambda d \lambda^{\prime} \rho(\lambda) \rho\left(\lambda^{\prime}\right) \log \left|\frac{\lambda-\lambda^{\prime}}{\Lambda}\right| .
$$

As a check of the choice of measure (similar to computations by Dijkgraaf and Vafa), we can take a superpotential of degree two, i.e. $W=\frac{1}{2} m \operatorname{Tr} \widehat{\Phi}^{2}$ and $m(\widehat{\Phi})_{f}^{\widetilde{f}}=\Lambda \delta_{f}^{\widetilde{f}}$. Explicit computation of the matrix integral (6.1) and (6.9) can be done. Both computations give the same answer,

$$
\mathcal{F}_{0}=\frac{1}{2} S^{2} \log \frac{m \Lambda^{2}}{S}+\frac{3}{4} S^{2} .
$$

For $\mathcal{F}_{1}$ we have,

$$
\mathcal{F}_{1}=\int d \lambda \rho(\lambda) \log \frac{B(\lambda)}{\Lambda^{N_{f}}} .
$$

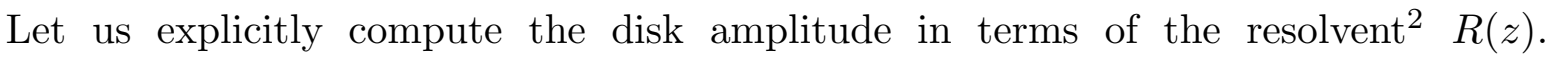
The integral in (6.11) can be written as a contour integral by recalling that $\rho(\lambda)=$ $-\left.\frac{1}{2 \pi i} \operatorname{disc} R(z)\right|_{z=\lambda}$

$$
\int d \lambda \rho(\lambda) \log B(\lambda)=\frac{1}{2 \pi i} \sum_{i=1}^{n} \oint_{A_{i}} d z R(z) \log B(z)
$$

2 Our definition of the resolvent differs by a sign from the standard matrix model convention (see for example [42]) which is given by $\frac{1}{\widehat{N}} \operatorname{Tr} \frac{1}{\widehat{\Phi}-z}$. 
In the $\log$ arithm we take the principal branch $\log r=\log |r|+i \alpha$ with $-\pi<\alpha \leq \pi$. Since $B(z)=B_{L} \prod_{I=1}^{L}\left(z-z_{I}\right)$ it has branch cuts which we take to run between $z_{I}$ and infinity ( $P$ in the upper sheet and $\widetilde{P}$ in the lower sheet). We make sure that they do not intersect the cuts of $R(z)$, such that (6.12) has no ambiguity.

The integral (6.12) can be written as

$$
\frac{1}{2 \pi i} \sum_{i=1}^{n} \oint_{A_{i}} d z R(z) \log B(z)=S \log B_{L}+\frac{1}{2 \pi i} \sum_{I=1}^{L} \sum_{i=1}^{n} \oint_{A_{i}} d z R(z) \log \left(z-z_{I}\right) .
$$

Now we deform the contour integral. We can do it either in the upper or the lower sheet. The simplest possibility is with $R(z)$ in the upper sheet because at infinity $R=$ $S / z+\mathcal{O}\left(z^{-2}\right)$. The contour integral is given by a sum of two terms. The first is an integral around infinity: a circle of radius $\left|\Lambda_{0}\right|$. This gives

$$
\frac{S L}{2 \pi i} \int_{\Lambda_{0}}^{e^{2 \pi i} \Lambda_{0}} \frac{\log z}{z} d z=S L \log \left(-\Lambda_{0}\right) .
$$

The second contribution is a sum of contour integrals around the cuts of $\log \left(z-z_{I}\right)$ which run from $q_{I}$ to $\Lambda_{0}$ :

$$
\frac{1}{2 \pi i} \sum_{I} \int_{C_{I}} d z R(z) \log \left(z-z_{I}\right)=-\sum_{I} \int_{q_{I}}^{\Lambda_{0}} R(z) d z
$$

The logarithmic divergences of the two contributions (6.14) and (6.15) cancel. This is not surprising, because the original contour integral (6.12) around the cuts of $R$ is finite.

We conclude that the disk amplitude is

$$
\mathcal{F}_{1}=S \log \left(\frac{\left(-\Lambda_{0}\right)^{L} B_{L}}{\Lambda^{N_{f}}}\right)-\sum_{I} \int_{q_{I}}^{\Lambda_{0}} R(z) d z
$$

We can also use $R(z)=\frac{1}{2}\left(W^{\prime}(z)-y(z)\right)$ to express the integral as an integral of $y$, and then we can write it as an integral in the second sheet

$$
\mathcal{F}_{1}=-\frac{1}{2} \sum_{I} \int_{\widetilde{q}_{I}}^{\widetilde{\Lambda}_{0}} y d z+S \log \left(\frac{\left(-\Lambda_{0}\right)^{L} B_{L}}{\Lambda^{N_{f}}}\right)-\frac{1}{2} L W\left(\Lambda_{0}\right)+\frac{1}{2} \sum_{I} W\left(z_{I}\right) .
$$




\section{Matrix Model Determination Of $M(z)$}

Before concluding this section, let us present a matrix model determination of (3.2). Recall the identification made in [27],

$$
\langle\widehat{M}(z)\rangle=\langle M(z)\rangle
$$

where $\langle M(z)\rangle$ is the field theory expectation value of the operator defined in (2.10) and $\langle\widehat{M}(z)\rangle$ is the average of the matrix function $\widehat{\widetilde{Q}} \frac{1}{z-\widehat{\Phi}} \widehat{Q}$. In order to compute the latter, we can first perform the gaussian integration over the matter fields $\widehat{\widetilde{Q}}$ and $\widehat{Q}$ in (6.1) for a given eigenvalue of $\widehat{\Phi}$. This leads to the insertion of $\frac{1}{\lambda_{k}-z} \frac{1}{m\left(\lambda_{k}\right)}$ in 6.7). In the saddle point approximation, we get $\langle\widehat{M}(z)\rangle$ by integrating the single eigenvalue result over the eigenvalue distribution, i.e.,

$$
\langle\widehat{M}(z)\rangle=\int d \lambda \frac{\rho(\lambda)}{\lambda-z} \frac{1}{m(\lambda)}
$$

Using that $\rho(\lambda)=-\left.\frac{1}{2 \pi i} \operatorname{disc} R(z)\right|_{z=\lambda}(6.19)$ can be written as,

$$
\langle\widehat{M}(z)\rangle=-\frac{1}{2 \pi i} \sum_{i=1}^{n} \oint_{A_{i}} \frac{R(x)}{x-z} \frac{1}{m(x)} d x
$$

which agrees with (3.2).

\section{The Off-Shell Effective Superpotential}

The field theory effective superpotential receives contributions from the sphere, $\mathcal{F}_{0}$, as well as from the disk, $\mathcal{F}_{1}$, amplitudes in the matrix model [27].

The sphere contribution up to terms with two derivatives of $S_{i}$ is

$$
\left.W_{\text {eff }}\right|_{\text {sphere }}=\sum_{i=1}^{n} N_{i} \frac{\partial \mathcal{F}_{0}}{\partial S_{i}}+2 \pi i \sum_{i=1}^{n-1} b_{i} S_{i}
$$

Note that no explicit $\tau_{0}^{f t} S$ appears in (7.1), where $\tau_{0}^{f t}$ is the bare coupling of the field theory. This is due to the way the matrix integral (6.1) is normalized. In this normalization, $\mathcal{F}_{0}$ is a finite quantity.

A detailed computation of $\frac{\partial \mathcal{F}_{0}}{\partial S_{i}}$ can be found in appendix B. The result is given by

$$
\frac{\partial \mathcal{F}_{0}}{\partial S_{i}}=-\frac{1}{2} \int_{\widehat{B}_{i}^{r}} y(z) d z+W\left(\Lambda_{0}\right)-2 S \log \left(-\frac{\Lambda_{0}}{\Lambda}\right)+\mathcal{O}\left(1 / \Lambda_{0}\right) .
$$


Therefore (7.1) is, up to $\mathcal{O}\left(1 / \Lambda_{0}\right)$ terms,

$$
\left.W_{\text {eff }}\right|_{\text {sphere }}=-\frac{1}{2} \sum_{i=1}^{n} N_{i} \int_{\widehat{B}_{i}^{r}} y(z) d z+N W\left(\Lambda_{0}\right)-2 N S \log \left(-\frac{\Lambda_{0}}{\Lambda}\right)+2 \pi i \sum_{i=1}^{n-1} b_{i} S_{i} .
$$

On the other hand, the disk contribution is

$$
\left.W_{\text {eff }}\right|_{\text {disk }}=\mathcal{F}_{1}
$$

Combining (7.3) with (6.17), we find that the effective superpotential of the theory is (up to terms with two derivatives of $\mathcal{F}_{0}$ and irrelevant $\mathcal{O}\left(1 / \Lambda_{0}\right)$ terms)

$$
\begin{aligned}
W_{\text {eff }}= & -\frac{1}{2} \sum_{i=1}^{n} N_{i} \int_{\widehat{B}_{i}^{r}} y(z) d z-\frac{1}{2} \sum_{I=1}^{L} \int_{\widetilde{q}_{I}}^{\widetilde{\Lambda}_{0}} y(z) d z \\
& +\frac{1}{2}(2 N-L) W\left(\Lambda_{0}\right)+\frac{1}{2} \sum_{I=1}^{L} W\left(z_{I}\right)-\pi i(2 N-L) S+2 \pi i \tau_{0} S+2 \pi i \sum_{i=1}^{n-1} b_{i} S_{i},
\end{aligned}
$$

where

$$
2 \pi i \tau_{0}=\log \left(\frac{B_{L} \Lambda^{2 N-N_{f}}}{\Lambda_{0}^{2 N-L}}\right) .
$$

This can be more conveniently written in terms of $R(z)=\frac{1}{2}\left(W^{\prime}(z)-y(z)\right)$ as follows:

$$
\begin{aligned}
W_{\text {eff }}= & \sum_{i=1}^{n} N_{i} \int_{\widehat{B}_{i}^{r}} R(z) d z+\sum_{I=1}^{L} \int_{\widetilde{q}_{I}}^{\widetilde{\Lambda}_{0}} R(z) d z \\
& +(N-L) W\left(\Lambda_{0}\right)+\sum_{I=1}^{L} W\left(z_{I}\right)-\pi i(2 N-L) S+2 \pi i \tau_{0} S+2 \pi i \sum_{i=1}^{n-1} b_{i} S_{i} .
\end{aligned}
$$

This superpotential is finite in the limit when $\Lambda_{0}$ and $\widetilde{\Lambda}_{0}$ are taken to $P$ and $\widetilde{P}$ respectively. All physical information should be computed in that limit. The logarithmic divergences in the contour integrals in (7.5) are cancelled by the $\Lambda_{0}$ dependence of $\tau_{0}$. Now we can identify the finite dimensionful parameter $\Lambda$ with the dynamical scale of the theory. Various special cases of this have been discussed in [6, 19, 23, 27]. The term with the integer constants $b_{i}$ was introduced in [34] and will be crucial below. The last two terms in (7.5) can be written as $\sum_{i} \oint_{A_{i}} y(z) d z \int_{\widehat{B}_{i}}\left(\bar{T}_{0} d \bar{z}+T_{0} d z\right)$ where $\bar{T}_{0} d \bar{z}+T_{0} d z$ (which we sometimes abbreviate simply as $\left.T_{0}\right)$ is a real differential whose $A_{i}$ and $\widehat{B}_{i}$ periods are $N_{i}$ and $-\tau_{0}-b_{i}$ respectively and which also has period minus one around $\widetilde{q}_{I}$. We will soon show that on shell $T$ and $T_{0}$ have the same periods. 
Here it is important to notice that, unlike cases studied in the literature, $\tau_{0}$ defined by (7.6) is not equal to the field theory bare coupling $\tau_{0}^{f t}$. In field theory, the one loop contribution to the superpotential implies that

$$
2 \pi i \tau_{0}^{f t}=\left(2 N-N_{f}\right) \log \left(\frac{\Lambda_{f t}}{\Lambda_{0}}\right)
$$

where $\Lambda_{f t}$ is the scale of the theory. Up to now, we have made the identification $\Lambda=\Lambda_{f t}$. Recall that $\Lambda$ was defined in the normalization of the matrix integral (6.1). If we relax this identification, a new term has to be added to the superpotential (7.1),

$$
\left.W_{\text {eff }}\right|_{\text {sphere }}=\sum_{i=1}^{n} N_{i} \frac{\partial \mathcal{F}_{0}}{\partial S_{i}}+\left(2 N-N_{f}\right) \log \left(\frac{\Lambda_{f t}}{\Lambda}\right) S+2 \pi i \sum_{i=1}^{n-1} b_{i} S_{i}
$$

Note that $W_{\text {eff }}$ in (7.9) is independent of $\Lambda$. Therefore, we can take $\Lambda=\Lambda_{0}$ and write,

$$
\left.W_{\text {eff }}\right|_{\text {sphere }}=\sum_{i=1}^{n} N_{i} \frac{\partial \mathcal{F}_{0}}{\partial S_{i}}+2 \pi i \tau_{0}^{f t} S+2 \pi i \sum_{i=1}^{n-1} b_{i} S_{i} .
$$

\section{Classical Limit Of $W_{\text {eff }}$}

A consistency check on (7.7) is to take the classical limit. In this limit the Riemann surface $\Sigma$ degenerates to $y^{2}=W^{\prime}(z)^{2}$. Therefore, $R(z) \rightarrow 0$ on the first sheet and $R(z) \rightarrow$ $W^{\prime}(z)$ on the second. In the pseudo-confining vacua, i.e., when all singularities of $T$ are on the second sheet, the first term in (7.7) gives $-N W\left(\Lambda_{0}\right)+\sum_{i=1}^{n} N_{i} W\left(a_{i}\right)$ while the second gives $L W\left(\Lambda_{0}\right)-\sum_{I=1}^{L} W\left(z_{I}\right)$. Combining this we get $\left.W_{\text {eff }}\right|_{c l}=\sum_{i=1}^{n} N_{i} W\left(a_{i}\right)$. On the other hand, Higgs vacua are obtained by moving some of the poles of $T$ at points $\widetilde{q}_{I}$ from the second to the first sheet. This has to be done through one of the cuts. Let us denote by $k_{i}$ the number of points on the first sheet obtained by moving the $\widetilde{q}_{I}$ through the $i^{t h}$ cut. In this case the contribution of the first term in (7.7) is unchanged but from the second we get,

$\sum_{I=1}^{L} r_{I} \int_{q_{I}}^{\widetilde{\Lambda}_{0}} R(z) d z+\sum_{I=1}^{L}\left(1-r_{I}\right) \int_{\widetilde{q}_{I}}^{\widetilde{\Lambda}_{0}} R(z) d z=L W\left(\Lambda_{0}\right)-\sum_{i=1}^{n} k_{i} W\left(a_{i}\right)-\sum_{I=1}^{L}\left(1-r_{I}\right) W\left(z_{I}\right)$.

Recall that $r_{I}$ is zero if the pole of $T$ at $z=z_{I}$ is on the second sheet and one if it is on the first sheet. 
Using this in (7.7) we get,

$$
\left.W_{e f f}\right|_{c l}=\sum_{i=1}^{n}\left(N_{i}-k_{i}\right) W\left(a_{i}\right)+\sum_{I=1}^{L} r_{I} W\left(z_{I}\right)
$$

\section{Field Equations Of $W_{\text {eff }}$}

We now proceed to the study of the field equations of the effective superpotential (7.5). Following [7], we replace the dynamical variables $S_{i}$ with the coefficients $f_{i}$ in $f(z)=$ $\sum_{i=1}^{n} f_{i} z^{i-1}$. Clearly $\frac{\partial}{\partial f_{i}} y d z=\frac{z^{i-1}}{2 y} d z$ are holomorphic differentials for $i=1, \ldots, n-1$. Linear combinations of them with coefficients which depend on $f_{i}$ are the holomorphic differentials $\zeta_{k}$. In addition to those we also have $g_{n} \frac{\partial}{\partial f_{n}} y d z=\frac{g_{n} z^{n-1}}{2 y} d z$. Where $g_{n}$ was introduced so that the differential has residue one and minus one at the poles of order one at $\widetilde{P}$ and $P$ respectively. Again, choosing appropriate linear combinations with the holomorphic differentials we get $\tau_{P, \widetilde{P}}$. Now consider the equation of motion of $f_{i}$ by varying (7.5) with respect to $f_{i}$. Since $W_{\text {eff }}$ is written as periods of $y d z$, the variation is expressed in terms of periods of the holomorphic differentials $\frac{z^{i-1}}{2 y} d z$ and the meromorphic differential $\frac{z^{n-1}}{2 y}$. Appropriate linear combinations of these give the following $n$ equations,

$$
\begin{aligned}
& 0=\sum_{i=1}^{n} N_{i} \int_{\widehat{B}_{i}} \zeta_{k}+\sum_{I=1}^{L} \int_{\widetilde{q}_{I}}^{\widetilde{P}} \zeta_{k}+b_{k} \\
& 0=\sum_{i=1}^{n} N_{i} \int_{\widehat{B}_{i}^{r}} \tau_{P, \widetilde{P}}+\sum_{I=1}^{L} \int_{\widetilde{q}_{I}}^{\widetilde{\Lambda}_{0}} \tau_{P, \widetilde{P}}-\log \frac{\Lambda_{0}^{2 N-L}}{B_{L} \Lambda^{2 N-N_{f}}}-\pi i(2 N-L) .
\end{aligned}
$$

We used that $\sum_{i=1}^{n} \oint_{A_{i}} \zeta_{k}=0, \oint_{A_{i}} \tau_{P, \widetilde{P}}=0$ for $i=1, \ldots, n-1$ and $\oint_{A_{i}} \zeta_{k}=\delta_{i k}$.

Comparing with (5.9) and (5.15) we see that these equations of motion are equivalent to

$$
\frac{1}{2 \pi i} \oint_{B_{i}} T(z) d z=-b_{i} \quad \int_{\widehat{B}_{n}^{r}} T(z) d z=-2 \pi i \tau_{0}=\log \frac{\Lambda_{0}^{2 N-L}}{B_{L} \Lambda^{2 N-N_{f}}} .
$$

Here $b_{i}$ 's are $n-1$ integers in the superpotential (7.5). Comparing (7.14) with (5.19) we find that $\Lambda^{2 N-N_{f}}$ in (7.14) differs by a factor of $2^{-N_{f} / 2}$ from $\Lambda^{2 N-N_{f}}$ in (5.19). This trivial factor can be reabsorbed in the definition of the normalization of the matrix integral (6.1). However, we have chosen not to include it in order to avoid cluttering the equations. 


\subsection{Alternative Proof}

\section{Preliminary Discussion}

In this subsection, we will present an alternative version of the proof that on-shell $T \equiv T(z) d z$ and $T_{0} \equiv T_{0}(z) d z$ are in the same cohomology class and in particular $T$ has integer periods. To make the ideas clear, we first present a simplified version in which we ignore the fact that some of the differential forms of interest have poles at infinity and also at the zeroes of the mass function $m(z)$. Then we incorporate the poles, first those at infinity and finally those due to $m(z)$.

So to begin with, we work on a closed Riemann surface $\Sigma$ of genus $g$, with a set of $A$-cycles $A_{i}$ and $B$-cycles $B_{i}, i=1, \ldots, g$. For any closed one-forms $\alpha$ and $\beta$ on $\Sigma$, we define

$$
I(\alpha, \beta)=\int_{\Sigma} \alpha \wedge \beta
$$

which we will usually write in the alternative form

$$
I(\alpha, \beta)=\sum_{i=1}^{g}\left(\oint_{A_{i}} \alpha \oint_{B_{i}} \beta-\oint_{A_{i}} \beta \oint_{B_{i}} \alpha\right) .
$$

As is clear from (7.15), $I(\alpha, \beta)=0$ if $\alpha$ and $\beta$ are both holomorphic differentials (in that case, locally $\alpha=f(z) d z, \beta=g(z) d z$, and $\alpha \wedge \beta=0$ ). There is a partial converse of this statement:

(A) If $\alpha$ is such that $I(\alpha, \beta)=0$ for any holomorphic differential $\beta$, then $\alpha$ is in the cohomology class of some holomorphic differential $\alpha^{\prime}$, and in particular $\alpha$ and $\alpha^{\prime}$ have the same periods.

The $A$-periods $\oint_{A_{i}} \alpha$ of a holomorphic one-form can be specified arbitrarily. But once this is done, the corresponding $B$-periods $\oint_{B_{i}} \alpha$ are uniquely determined. The formula expressing the $B$-periods in terms of the $A$-periods involves the "period matrix" of $\Sigma$, and depends on the complex moduli of $\Sigma$. In our problem, even off-shell, that is, before we fix the complex moduli of $\Sigma$, the $A$-periods of $T$ are equal to integers $N_{i}$ that depend on the choice of a symmetry-breaking pattern. We have the following statement even off-shell:

(B) For any choice of moduli of $\Sigma$, the differential $T$ is uniquely determined by being a holomorphic differential with $\frac{1}{2 \pi i} \oint_{A_{i}} T=N_{i}$.

If we combine statements $(A)$ and $(B)$, we arrive at the following:

(C) The cohomology class of a closed one-form $T$ is uniquely determined by the assertions that $(i) I(T, \omega)=0$ for any holomorphic one-form $\omega$; (ii) $\frac{1}{2 \pi i} \oint_{A_{i}} T=N_{i}$. 
If, therefore, $T_{0}$ is any differential form that obeys the same conditions, then $T$ and $T_{0}$ have the same periods.

In the simplified problem that we will consider first, we take the superpotential to be

$$
W=\sum_{i} N_{i} \oint_{B_{i}} \gamma+\sum_{i} b_{i} \oint_{A_{i}} \gamma,
$$

where $\gamma$ is the meromorphic differential $\gamma=R(z) d z$, while $N_{i}$ and $b_{i}$ are integers. We can write

$$
W=\frac{1}{2 \pi i} I\left(T_{0}, \gamma\right),
$$

where $T_{0}$ is a closed (not necessarily holomorphic) one-form with

$$
\frac{1}{2 \pi i} \oint_{A_{i}} T_{0}=N_{i}, \quad \frac{1}{2 \pi i} \oint_{B_{i}} T_{0}=-b_{i} .
$$

We also assume that $\gamma$ has the special property that, with $f_{i}$ being the moduli of $\Sigma$,

$$
\frac{\partial \gamma}{\partial f_{i}}=\omega_{i}
$$

with $\omega_{i}$ being a basis of the space of holomorphic differentials on $\Sigma$. (In our actual problem, this is almost true, except that one of the $\omega_{i}$ has a pole at infinity. As noted above, we will postpone incorporating the poles until we have explained the structure of the argument.)

Using (7.18) and (7.20), the condition for a critical point of $W$, which is $\partial W / \partial f_{i}=0$, becomes $I\left(T_{0}, \omega_{i}\right)=0$. But given our assumption that the $\omega_{i}$ are a basis of the space of holomorphic differentials on $\Sigma$, this condition says exactly that $I\left(T_{0}, \omega\right)=0$ for any holomorphic differential $\omega$. In other words, on-shell $T_{0}$ obeys condition (i) in statement (C). Since $T_{0}$ also obeys condition (ii) by virtue of its definition (7.19), it follows as stated in $(C)$ that $T$ and $T_{0}$ have the same cohomology class on-shell. In particular, on-shell $\frac{1}{2 \pi i} \oint_{B_{i}} T=-b_{i}$.

\section{Incorporation Of Poles At Infinity}

Our goal now, as promised above, is to describe an argument with the same structure, reaching the same conclusion, but properly incorporating the poles that are present in some of the differential forms in our actual problem.

In particular, in our problem, the differential form $\gamma=R(z) d z$ has a pole of order $n$ at the points $P$ and $\widetilde{P}$ that correspond to $z=\infty$ on the two sheets of $\Sigma$. The singularities of $\omega_{i}=\partial \gamma / \partial f_{i}$ are milder; the $\omega_{i}$ have at worst simple poles at $P$ and $\widetilde{P}$. 
Likewise, $T$ has simple poles at $P$ and $\widetilde{P}$. If we include fundamental matter, then $T$ has additional poles, as we have seen in section 4 ; for the moment, we consider the problem without fundamental matter.

The existence of all of these poles means that the argument we have presented above does not quite fit our physical problem. It also means that some regularization is required in the definition of the superpotential. We are going to pick a regularization which is such that the proof of the on-shell behavior of $T$ will go through directly for the regularized theory, without having to take the regulator to infinity.

Our method of regularization in this section will be to pick points $\Lambda_{0}$ and $\widetilde{\Lambda}_{0}$ that are "near" $P$ and $\widetilde{P}$ and to place the poles of $T$ at $\Lambda_{0}$ and $\widetilde{\Lambda}_{0}$ rather than at $P$ and $\widetilde{P}$. We take $\Lambda_{0}$ and $\widetilde{\Lambda}_{0}$ to lie above the same (large) value of $z$ on the two different sheets; later we will keep fixed this value while varying the moduli of the hyperelliptic curve $y^{2}=W^{\prime}(z)^{2}+f(z)$. It is important to note that in this regularization, $T$ is a solution to the anomaly equations up to terms of order $1 / \Lambda_{0}$. By contrast, in the regulatization of section $5, T$ was a solution to the anomaly equations before taking the cut off to infinity.

Now let us explain the analogs of some assertions that we made in our preliminary discussion. Let $\Sigma^{\prime}$ be the Riemann surface with punctures obtained by deleting $\Lambda_{0}$ and $\widetilde{\Lambda}_{0}$ from the closed Riemann surface $\Sigma$. Let $\alpha$ be a closed one-form on $\Sigma^{\prime}$. Then if $A_{\Lambda}$ is a small contour circling once around $\Lambda_{0}$ in the counterclockwise direction, we may have $\oint_{A_{\Lambda}} \alpha \neq 0$. Likewise, let $\Sigma^{\prime \prime}$ be obtained by deleting from $\Sigma$ the punctures $P$ and $\widetilde{P}$, and let $\beta$ be a closed one-form on $\Sigma^{\prime \prime}$. Then if $A_{P}$ is a small contour circling once around $P$ in the counterclockwise direction, we may have $\oint_{A_{P}} \beta \neq 0$. We also let $B_{\Lambda}$ be a path from $\widetilde{\Lambda}_{0}$ to $\Lambda_{0}$ (avoiding $P$ and $\widetilde{P}$ ), and $B_{P}$ a path from $\widetilde{P}$ to $P$ (avoiding $\Lambda_{0}$ and $\widetilde{\Lambda}_{0}$ ). $\alpha$ has a well-defined integral over $B_{P}$, and $\beta$ has a well-defined integral over $B_{\Lambda}$, but not vice-versa. We also let $A_{i}$ and $B_{i}, i=1, \ldots, g$ be a complete set of compact $A$ - and $B$-cycles on the genus $g$ surface $\Sigma$, chosen to avoid the points $P, \widetilde{P}, \Lambda_{0}$, and $\widetilde{\Lambda}_{0}$. In the problem studied in this paper, $g=n-1$. We make the above choices so that $B_{P}$ and $B_{\Lambda}$ have vanishing intersection numbers with the compact cycles. This condition identifies $B_{P}$ with $B_{n}$ and $B_{\Lambda}$ with the regularized version of $\widehat{B}_{n}$, i.e., $\widehat{B}_{n}^{r}$, as defined in section 5 .

Now we define

$$
I(\alpha, \beta)=\oint_{A_{\Lambda}} \alpha \int_{B_{\Lambda}} \beta-\oint_{A_{P}} \beta \int_{B_{P}} \alpha+\sum_{i=1}^{g}\left(\oint_{A_{i}} \alpha \oint_{B_{i}} \beta-\oint_{A_{i}} \beta \oint_{B_{i}} \alpha\right) .
$$


The notation $A_{P}, A_{\Lambda}$ and also $B_{P}, B_{\Lambda}$ is meant to suggest that one can fruitfully think of $A_{P}, A_{\Lambda}$ as extra $A$-cycles (for $\beta$ and $\alpha$, respectively), and $B_{P}, B_{\Lambda}$ as extra $B$-cycles.

Finally, by an allowed holomorphic differential on $\Sigma^{\prime \prime}$, we will mean a holomorphic one-form on $\Sigma^{\prime \prime}$ that has at worst simple poles at the points $P$ and $\widetilde{P}$. Similarly, an allowed holomorphic differential on $\Sigma^{\prime}$ has at worst simple poles at $\Lambda_{0}$ and $\widetilde{\Lambda}_{0}$. .

The Riemann bilinear relations (see eqn. A.9) says that if $\alpha$ and $\beta$ are allowed holomorphic differentials on $\Sigma^{\prime}$ and $\Sigma^{\prime \prime}$, respectively, then $I(\alpha, \beta)=0.5$ Moreover, just as in the absence of poles, this statement has a partial converse:

$\left(A^{\prime}\right)$ If $\alpha$ is such that $I(\alpha, \beta)=0$ for any allowed holomorphic differential $\beta$, then $\alpha$ is in the same cohomology class (and has the same integral over $B_{P}$ ) as some allowed holomorphic differential $\alpha^{\prime}$, and in particular $\alpha$ and $\alpha^{\prime}$ have the same periods.

The $A$-periods $\oint_{A_{i}} \alpha$ of a holomorphic one-form $\alpha$ on $\Sigma^{\prime}$ can be specified arbitrarily, along with the value of $\oint_{A_{\Lambda}} \alpha$. But once this is done, the corresponding $B$-periods $\oint_{B_{i}} \alpha$ are uniquely determined, along with $\int_{B_{P}} \alpha$. The formula expressing the $B$-periods in terms of the $A$-periods involves a generalized "period matrix" of $\Sigma$, and depends on the complex moduli of $\Sigma$. In our problem, even off-shell, that is, before we fix the complex moduli of $\Sigma$, the $A$-periods of $T$ are equal to integers $N_{i}$ that depend on the choice of a symmetry-breaking pattern, and we require in addition that $\frac{1}{2 \pi i} \oint_{A_{\Lambda}} T=N$ (this being the regularized version of the assertion that $T$ has a pole at infinity with residue $N$ ). We have the following statement even off-shell:

$\left(B^{\prime}\right)$ For any choice of moduli of $\Sigma$, the differential $T$ is uniquely determined by being an allowed holomorphic differential on $\Sigma^{\prime}$ with $\frac{1}{2 \pi i} \oint_{A_{i}} T=N_{i}, \frac{1}{2 \pi i} \oint_{A_{\Lambda}} T=N$.

If we combine statements $\left(A^{\prime}\right)$ and $\left(B^{\prime}\right)$, we arrive at the following:

$\left(C^{\prime}\right)$ The cohomology class of a closed one-form $T$ on $\Sigma^{\prime}$ is uniquely determined by the assertions that (i) $I(T, \omega)=0$ for any allowed holomorphic one-form $\omega$ on $\Sigma^{\prime \prime}$; (ii)

3 Indeed, if one glues $P$ to $\widetilde{P}$ and $\Lambda_{0}$ to $\widetilde{\Lambda}_{0}$, then after smoothing the resulting singularities, one gets a smooth surface $\widehat{\Sigma}$ of genus $g+2$, with $A_{P}$ and $A_{\Lambda}$ as additional $A$-cycles and $B_{P}, B_{\Lambda}$ as additional $B$-cycles.

4 An allowed holomorphic differential is precisely one that corresponds to the limit of an ordinary holomorphic differential on the genus $g+2$ surface $\widehat{\Sigma}$ of the last footnote.

5 This assertion can be deduced from the fact that $I(\alpha, \beta)=0$ for ordinary holomorphic differentials $\alpha, \beta$ on $\widehat{\Sigma}$, where here as $\widehat{\Sigma}$ is smooth and $\alpha$ and $\beta$ are assumed to have no poles on $\widehat{\Sigma}$, we use the original definition $(\overline{7.15})$ of $I(\alpha, \beta)$. 
$\frac{1}{2 \pi i} \oint_{A_{i}} T=N_{i}$ and $\frac{1}{2 \pi i} \oint_{A_{\Lambda}} T=N$. If, therefore, $T_{0}$ is any differential form that obeys the same conditions, then $T$ and $T_{0}$ have the same periods.

Now, we let $T_{0}$ be a closed one-form on $\Sigma^{\prime}$ with

$$
\begin{aligned}
& \frac{1}{2 \pi i} \oint_{A_{i}} T_{0}=N_{i} \\
& \frac{1}{2 \pi i} \oint_{A_{\Lambda}} T_{0}=N \\
& \frac{1}{2 \pi i} \oint_{B_{i}} T_{0}=-b_{i} \\
& \frac{1}{2 \pi i} \int_{B_{P}} T_{0}=-\tau_{0} .
\end{aligned}
$$

Here $\tau_{0}$ is the bare coupling of the gauge theory.

We define the regularized superpotential as

$$
W=\frac{1}{2 \pi i} I\left(T_{0}, \gamma\right)
$$

where $\gamma$ is the familiar differential $\gamma=R(z) d z \cdot \gamma$ is holomorphic on $\Sigma^{\prime \prime}$, but it is not what we have called an allowed holomorphic differential, as it has poles at $P$ and $\widetilde{P}$ of order greater than one. Nevertheless, the expression $I\left(T_{0}, \gamma\right)$ is completely well-defined; for its definition, it suffices that $T_{0}$ be regular on $\Sigma^{\prime}$ and $\gamma$ on $\Sigma^{\prime \prime}$, regardless of the nature of the behavior near the respective punctures.

Just as before, if $f_{i}$ are the complex moduli of $\Sigma$, then the equation for a critical point of $W$ is $I\left(T_{0}, \omega_{i}\right)=0$ where $\omega_{i}=\partial \gamma / \partial f_{i}$. Unlike $\gamma$, the $\omega_{i}$ have only simple poles at the punctures and so are allowed holomorphic differentials; conversely, any allowed holomorphic differential on $\Sigma^{\prime \prime}$ is a linear combination of the $\omega_{i}$. So the condition for a critical point of $W$ is precisely that $T_{0}$ obeys condition $(i)$ in statement $\left(C^{\prime}\right)$. Since $T_{0}$ was also defined (in (7.22) ) to obey condition (ii), it follows from statement $\left(C^{\prime}\right)$ that on-shell, in other words when the moduli of $\Sigma$ are chosen to get a critical point of $W, T_{0}$ and $T$ have the same cohomology class. In particular, on-shell, $\frac{1}{2 \pi i} \oint_{B_{i}} T=-b_{i}$ and the periods of $T$ on compact cycles are all integers.

\section{Inclusion Of Fundamental Matter}

We developed in section 4 the necessary facts for including fundamental matter in this discussion. Including fundamental matter means that $T$ has simple poles, with residue 1, at certain points $U_{I}$ on $\Sigma$. (The $U_{I}$ may lie on the first or second sheet; this choice will 
not affect our analysis and will not be built into the notation.) $\gamma$ does not have additional poles. So in defining $\Sigma^{\prime}$, we omit the $U_{I}$ as well as $\Lambda_{0}$ and $\widetilde{\Lambda}_{0}$, while in defining $\Sigma^{\prime \prime}$, we omit only the original punctures $P$ and $\widetilde{P}$. An allowed holomorphic differential on $\Sigma^{\prime}$ or $\Sigma^{\prime \prime}$ has only simple poles at the punctures.

We let $A_{I}$ be a small contour circling $U_{I}$ once in the counterclockwise direction, and $B_{I}$ a path from $U_{I}$ to $P$ avoiding all other punctures, and with vanishing intersection with all the other cycles. For $\alpha$ a closed one-form on $\Sigma^{\prime}$ and $\beta$ a closed one-form on $\Sigma^{\prime \prime}$, we define

$I(\alpha, \beta)=\oint_{A_{\Lambda}} \alpha \int_{B_{\Lambda}} \beta+\sum_{I} \oint_{A_{I}} \alpha \int_{B_{I}} \beta-\oint_{A_{P}} \beta \int_{B_{P}} \alpha+\sum_{i=1}^{g}\left(\oint_{A_{i}} \alpha \oint_{B_{i}} \beta-\oint_{A_{i}} \beta \oint_{B_{i}} \alpha\right)$.

All previous statements have obvious analogs. If $\alpha$ and $\beta$ are, respectively, allowed holomorphic differentials on $\Sigma^{\prime}$ and $\Sigma^{\prime \prime}$, then $I(\alpha, \beta)=0$. This statement has the partial converse stated as $\left(A^{\prime}\right)$ above. We will omit copying this statement.

Moreover, $\left(B^{\prime}\right)$ and $\left(C^{\prime}\right)$ have obvious analogs. The $A$-periods $\oint_{A_{i}} \alpha$ of a holomorphic one-form $\alpha$ on $\Sigma^{\prime}$ along with the values of $\oint_{A_{\Lambda}} \alpha$ and $\oint_{A_{I}} \alpha$ can be specified arbitrarily. Once this is done, the corresponding $B$-periods $\oint_{B_{i}} \alpha$ are uniquely determined along with $\int_{B_{P}} \alpha$, and depend on the moduli of $\Sigma$. However:

$\left(B^{\prime \prime}\right)$ For any choice of moduli of $\Sigma$, the differential $T$ is uniquely determined by being an allowed holomorphic differential on $\Sigma^{\prime}$ with $\frac{1}{2 \pi i} \oint_{A_{i}} T=N_{i}, \frac{1}{2 \pi i} \oint_{A_{\Lambda}} T=N$, $\frac{1}{2 \pi i} \oint_{A_{I}} T=1$.

If we combine statements $\left(A^{\prime}\right)$ and $\left(B^{\prime \prime}\right)$, we arrive at the following:

$\left(C^{\prime \prime}\right)$ The cohomology class of a closed one-form $T$ on $\Sigma^{\prime}$ is uniquely determined by the assertions that $(i) I(T, \omega)=0$ for any allowed holomorphic one-form $\omega$ on $\Sigma^{\prime \prime}$; (ii) $\frac{1}{2 \pi i} \oint_{A_{i}} T=N_{i}, \frac{1}{2 \pi i} \oint_{A_{\Lambda}} T=N$, and $\frac{1}{2 \pi i} \oint_{A_{I}} T=1$. If, therefore, $T_{0}$ is any differential form that obeys the same conditions, then $T$ and $T_{0}$ have the same periods.

The rest of the argument is hopefully clear. We let $T_{0}$ be a closed one-form on $\Sigma^{\prime}$ with

$$
\begin{aligned}
& \frac{1}{2 \pi i} \oint_{A_{i}} T_{0}=N_{i} \\
& \frac{1}{2 \pi i} \oint_{A_{\Lambda}} T_{0}=N \\
& \frac{1}{2 \pi i} \oint_{A_{I}} T_{0}=1 \\
& \frac{1}{2 \pi i} \oint_{B_{i}} T_{0}=-b_{i} \\
& \frac{1}{2 \pi i} \int_{B_{P}} T_{0}=-\tau_{0} .
\end{aligned}
$$


We define the superpotential as $W=\frac{1}{2 \pi i} I\left(T_{0}, \gamma\right)$. The condition for a critical point is, exactly as before, that $I\left(T_{0}, \omega\right)=0$ for every allowed holomorphic differential $\omega$ on $\Sigma^{\prime \prime}$. Hence on-shell, $T_{0}$ obeys the conditions in $\left(C^{\prime \prime}\right)$. Therefore, $T$ and $T_{0}$ have the same periods and in particular $\frac{1}{2 \pi i} \oint_{B_{i}} T=-b_{i}$.

The superpotential $W_{\text {eff }}=\frac{1}{2 \pi i} I\left(T_{0}, \gamma\right)$ has been characterized so far by conditions on its critical points. This still allows for an additive piece independent of the complex structure of the Riemann surface $\Sigma$. In order to fix this piece, consider the classical limit in which the Riemann surface degenerates to $y^{2}=W^{\prime}(z)^{2}$. This leads to $R(z)=0$ on the first sheet and $R(z)=W^{\prime}(z)$ on the second. Recalling that $\gamma=R(z) d z$ and the conditions (7.25), we get

$$
\left.\frac{1}{2 \pi i} I\left(T_{0}, \gamma\right)\right|_{c l}=\sum_{i=1}^{n} N_{i} W\left(a_{i}\right)+(L-N) W\left(\Lambda_{0}\right)-\sum_{I=1}^{L} W\left(z_{I}\right) .
$$

On the other hand the classical superpotential should be $\left.W_{\text {eff }}\right|_{c l}=\sum_{i=1}^{n} N_{i} W\left(a_{i}\right)$. Therefore,

$$
W_{e f f}=\frac{1}{2 \pi i} I\left(T_{0}, \gamma\right)+(N-L) W\left(\Lambda_{0}\right)+\sum_{I=1}^{L} W\left(z_{I}\right)
$$

In order to compare this superpotential with $(7.7)$, we should recall that the regularization procedure is different. The only place this is relevant is in the integral of $T$ along $B_{P}$ and the $B_{I}$ 's. $T$ has a pole at $\Lambda_{0}$ and $\widetilde{\Lambda}_{0}$. However, the $T$ solution to the anomaly equations has a pole at $P$ and $\widetilde{P}$. The difference of the integrals can be shown to be equal to half the period of $T$ around $A_{\Lambda}$ up to terms of order $1 / \Lambda_{0}$. This implies that the difference is equal to $\pi i$. This accounts for the $\pi i(L-2 N) S$ term in $(7.7)$.

\section{Interpolating Between Different Vacua}

We start this discussion by considering a certain process involving variation of the parameters in our model. We consider an example that can be understood semiclassically.

Let as usual $a_{j}, j=1, \ldots, n$ be the zeroes of $W^{\prime}(z)$, and $z_{I}$ the zeroes of the mass function $B(z)=\operatorname{det} m(z)$. We consider a classical vacuum with $N_{j}$ eigenvalues of $\Phi$ set equal to $a_{j}$, leaving a $U\left(N_{j}\right)$ factor in the low energy gauge group. Now consider a process in which, keeping the other parameters fixed, one of the $z_{I}$ circles counterclockwise once around one particular $a_{i}$ in the complex plane, chosen so that $N_{i} \neq 0$. Since $z_{I}$ determines one of the quark mass parameters in the effective $U\left(N_{i}\right)$ theory, in this process one of those parameters changes in phase by $2 \pi$. For example, if $N_{f}=1$ and $m(z)=z_{I}-z$, then the quark mass parameter is $z_{I}-a_{i}$, and changes in phase by $2 \pi$ when $z_{I}$ circles around $a_{i}$. In the process, the theta angle $\theta_{i}$ of the $U\left(N_{i}\right)$ theory increases by $2 \pi$. 

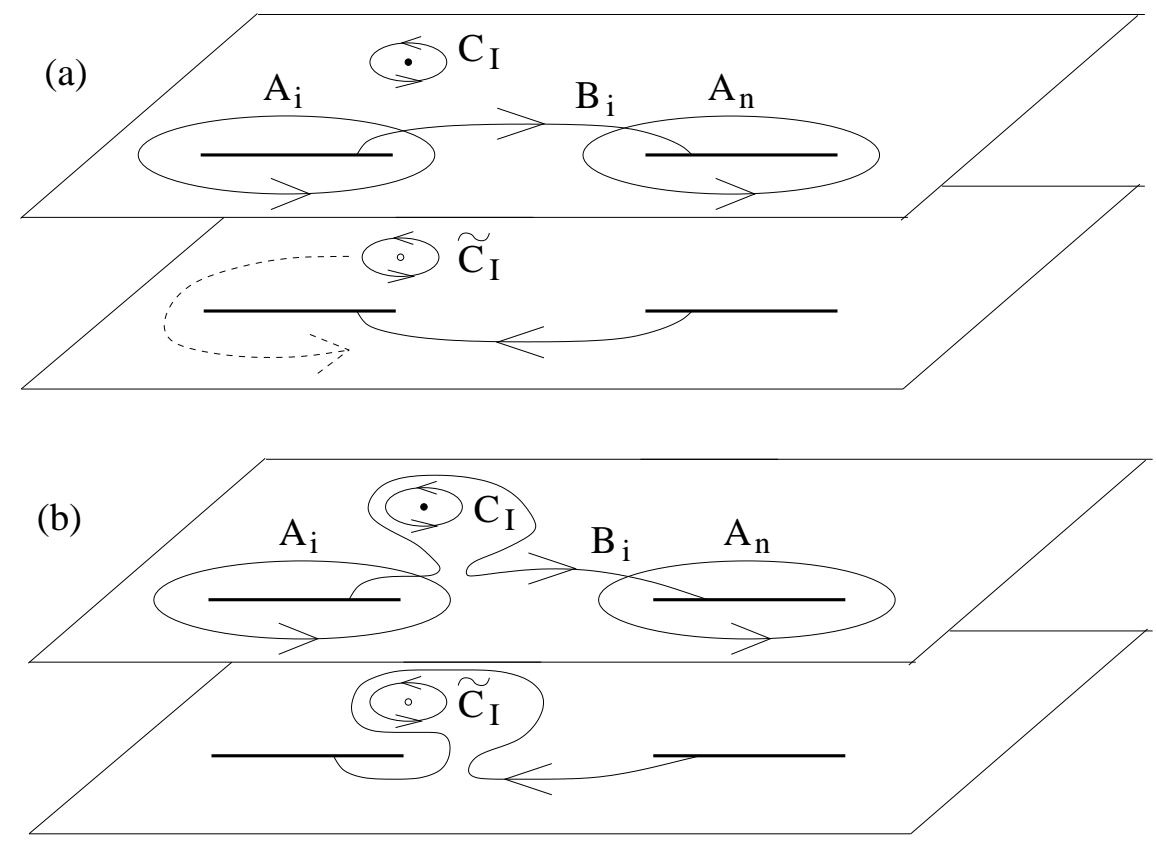

Fig. 3: Interpolation between different $\theta_{i}$ values. (a) Original configuration. Cycle $B_{i}$ is shown. On the upper sheet it is oriented from the $i^{t h}$ cut to the $n^{\text {th }}$ cut while on the lower sheet from the $n^{\text {th }}$ cut to the $i^{\text {th }}$ cut. The points $q_{I}$ (black dot) and $\widetilde{q}_{I}$ (white dot) are enclosed by the $C_{I}$ and $\widetilde{C}_{I}$ contours respectively. The dashed line represents the motion in the lower sheet of $\widetilde{q}_{I}$ as it circles once around the $i^{\text {th }}$ cut. $q_{I}$ also moves for $z\left(q_{I}\right)=z\left(\widetilde{q}_{I}\right)$. (b) Final configuration after the points $\widetilde{q}_{I}$ and $q_{I}$ return to their original position completing the trajectory around the $i^{\text {th }}$ cut. Also shown is the deformation of the original $B_{i}$ cycle. Clearly, the new $B_{i}$ cycle is given by $B_{i}-\widetilde{C}_{I}+C_{I}$.

The periods of $T(z) d z$ integrated over certain cycles measure differences between theta angles [34]. In particular, if the $B_{i}$ are the compact cycles described in figure 2, and we set

$$
b_{i}=-\frac{1}{2 \pi i} \oint_{B_{i}} T(z) d z
$$

then we have

$$
\theta_{i}-\theta_{n}=2 \pi b_{i}
$$

When $z_{I}$ circles around $a_{i}$ and $\theta_{i} \rightarrow \theta_{i}+2 \pi$, we must therefore have

$$
b_{i} \rightarrow b_{i}+1
$$

We could also have obtained the last formula by an argument similar to that used in sections 2 and 4 to explain the change in the $N_{i}$ when $z_{I}$ passes through a cut. We will carry out the discussion first in the pseudo-confining phase with $r_{I}=0$. For any given $z_{I}$, 
the differential $T(z) d z$ has a pole of residue 1 at the point $\widetilde{q}_{I}$ corresponding to $z_{I}$ on the second sheet. When $z_{I}$ migrates around $a_{i}, \widetilde{q}_{I}$ sweeps out a closed path $\tilde{Y}$ that intersects the cycle $B_{i}$. To avoid the singularity, one should move the cycle $B_{i}$ continuously in (8.1) as $z_{I}$ is moved. By the time $z_{I}$ returns to its starting point, $B_{i}$ returns as $B_{i}-\widetilde{C}_{I}$, where $\widetilde{C}_{I}$ is a small contour that wraps once around $\widetilde{q}_{I}$. Since $\frac{1}{2 \pi i} \oint_{C_{I}} T(z) d z=1$, this leads to $(8.3)$.

What happens if we consider the same motion of $z_{I}$, but this time in the Higgs phase, in which the pole associated with $z_{I}$ is on the first sheet at $q_{I}$ (rather than on the second sheet at $\left.\widetilde{q}_{I}\right)$ ? In this case, as $z_{I}$ migrates around $a_{i}, q_{I}$ sweeps out a closed path $Y$ which intersects the contour $B_{i}$, but now, since the part of the contour $B_{I}$ that is on the first sheet runs in the opposite direction (if projected to the $z$-plane) from that in the second sheet, the sign of the intersection number is reversed,

$$
Y \cap B_{i}=-\tilde{Y} \cap B_{i}
$$

To avoid the singularity, the cycle $B_{i}$ must again be varied continuously; it returns as $B_{i}+C_{I}$, where $C_{I}$ is a small contour surrounding $q_{I}$. We get $B_{i}+C_{I}$, compared to $B_{i}-\widetilde{C}_{I}$ in the last paragraph, because of the relative minus sign in (8.4). The opposite sign implies that in the Higgs phase, with $r_{I}=1$, the shift in $b_{i}$ when $z_{I}$ migrates around $a_{i}$ is $b_{i} \rightarrow b_{i}-1$, compared to $b_{i} \rightarrow b_{i}+1$ in the last paragraph. The sign reversal can be readily explained in the field theory. For $r_{I}=0$, as $z_{I} \rightarrow a_{i}$, components of $Q$ and $\widetilde{Q}$ comprising a chiral multiplet in the fundamental representation of $U\left(N_{i}\right)$ (plus its conjugate) become massless; their contribution to the $U\left(N_{i}\right)$ beta function is +1 . For $r_{I}=1$, as $z_{I} \rightarrow a_{i}$, the expectation value of the adjoint superfield $\Phi$ as well as $Q$ and $\widetilde{Q}$ change in such a way (see (2.6)) that the unbroken $U\left(N_{i}\right)$ is extended to an unbroken $U\left(N_{i}+1\right)$. The extra fields that become massless consist of a vector multiplet in the fundamental representation (the gauge fields that extend $U\left(N_{i}\right)$ to $U\left(N_{i}+1\right)$ ) along with a chiral multiplet (coming from $Q)$ plus similar multiplets in the conjugate representation. The total contribution to the beta function of these fields is $-3+1=-2$, where -3 is the contribution of the vector multiplet. The opposite sign beta function determines the opposite sign contribution to $b_{i}$ from integrating out these multiplets near $a_{i} \approx z_{I}$. In getting the monodromy of the theta angle, the 2 in the beta function is cancelled by the fact that, according to (2.7), the expectation value of $Q$ (and hence the masses produced by the Higgs mechanism) are proportional to $\sqrt{z_{I}-a_{i}}$. More explicitly, the instanton factor of the low energy gauge 
group $\Lambda_{L}^{p}$ depends on the beta function. Near $a_{i} \approx z_{I}$ it scales like $\Lambda_{L}^{p} \sim z_{I}-a_{i}$ for $r_{I}=0$ and $\Lambda_{L}^{p} \sim\left(z_{I}-a_{i}\right)^{-1}$ for $r_{I}=1$. Therefore depending on whether $r_{I}=0$ or $r_{I}=1, b_{i}$ changes by opposite amounts when $z_{I}$ winds around $a_{i}$.

We can express the result (8.3) in the following fashion. While $z_{I}$ is circling around $a_{i}$, the position $\widetilde{q}_{I}$ of the pole in $T$ associated with $z_{I}$ migrates around a certain closed path $C$ on our Riemann surface $\Sigma$. $C$ has intersection number 1 with the closed contour $B_{i}$, as indicated in the figure 3. The transformation law of $b_{i}$ is $b_{i} \rightarrow b_{i}+B_{i} \cap C$, where $B_{i} \cap C$ is the intersection number of $B_{i}$ and $C$. We can state this more invariantly by saying that for any closed contour $D$ in $\Sigma$, when a pole of $T$ passes around a contour $C$, the period of $T$ changes by

$$
\frac{1}{2 \pi i} \oint_{D} T(z) d z \rightarrow \frac{1}{2 \pi i} \oint_{D} T(z) d z+D \cap C .
$$

In fact, of the $A_{j}$ and $B_{j}$ cycles in figure 2 , only $B_{i}$ has a nonzero intersection number with $C$; and only the period $b_{i}=-\frac{1}{2 \pi i} \oint_{B_{i}} T(z) d z$ is expected to change in a semiclassical process in which $z_{I}$ circles around one of the $a_{i}$. An even more invariant way to say this is that the cohomology class of $\frac{1}{2 \pi i} T(z) d z$ changes in such a process by $\frac{1}{2 \pi i} T(z) d z \rightarrow$ $\frac{1}{2 \pi i} T(z) d z+[C]$, where $[C]$ is the cohomology class that is Poincaré dual to $C$.

In section 4 , we considered a process in which one of the $z_{I}$ (or more precisely the corresponding $q_{I}$ ) passes through the cut $A_{i}$ onto the second sheet. We showed that in this process, there is a transition from a pseudo-confining phase to a Higgs phase, and a transformation $N_{i} \rightarrow N_{i}-1$. Consider now the following process that starts and ends at the same phase, which we can take to be pseudo-confining: we let $q_{I}$ start on the first sheet, pass through the cut $A_{i}$ to the second sheet, and then return to the first sheet by passing through the cut $A_{i^{\prime}}$. Clearly, in this process the $N$ 's transform by $N_{i} \rightarrow N_{i}-1$, $N_{i^{\prime}} \rightarrow N_{i^{\prime}}+1$, with no change in the others. This can be regarded as another illustration of eqn. (8.5). In this case, one of the poles of $T$ has made a closed circuit $C$ from the second sheet to the first and back again; of the canonical $A_{j}$ and $B_{j}$ cycles of figure 2 , the ones that have non-zero intersection with $C$ are $A_{i}$ and $A_{i^{\prime}}$.

Classically, the pseudo-confining phases are labeled by the integers $N_{i}$ with the restriction $\sum_{i} N_{i}=N$, as well as by the $b_{i}$. By iterating the processes that we have so far described, we can (with a caveat explained shortly) change in an arbitrary fashion the $N_{i}$ and $b_{i}$, so these operations connect all of the pseudo-confining phases. By combining these operations with a further process in which some $q_{I}$ move to the second sheet and stay there, 
we can similarly connect pseudo-confining phases to arbitrary Higgs phases. The ability to connect all the phases in this way should not come as a complete surprise, because in this model, in contrast to that studied in [34], the presence of fields in the fundamental representations prevents the existence of precise order parameters involving Wilson and 't Hooft loops of the underlying $U(N)$ gauge theory.

\section{On-Shell And Off-Shell Continuation}

This discussion is subject to an important caveat. As in section 4, we have here imagined changing $m(z)$ while keeping fixed the Riemann surface $\Sigma$. But $\Sigma$ depends on the resolvent $R(z)$ and thus on the gluino condensates $S_{i}$; on-shell, the $S_{i}$ should really be determined by extremizing the effective superpotential $W_{\text {eff }}$, and $W_{\text {eff }}$ depends on $m(z)$. So in general, when $m(z)$ is changed, the $S_{i}$ and $\Sigma$ will change. A more delicate question now arises: if while changing $m(z)$, one adjusts the $S_{i}$ so as to remain on-shell, is the continuation between the different phases still possible?

The process described involving a change in the $b_{i}$ when the $z_{I}$ circle around the $a_{i}$ can be seen semiclassically and so can certainly occur on-shell. The process in which $q_{I}$ passes through a cut, changing some $N_{i}$, is more delicate as the region with $q_{I}$ near the cut is strongly coupled. Intuitively, we believe that when the $N_{i}$ are large enough, the back-reaction due to a single eigenvalue passing through the cut is small, and hence it should be possible for all of these processes to occur on-shell. A bit of evidence in this direction is that for $\mathcal{N}=2$ super Yang-Mills theory with $S U(2)$ gauge group and one flavor, perturbed slightly to $\mathcal{N}=1$ by a mass for the adjoint superfield, it is possible to have a smooth transition from a branch with a condensed monopole and "confinement" to a branch with a condensed quark and a "Higgs effect" [37]. The model is a special case (with $N=2, N_{f}=1$, linear $m(z)$, and quadratic $W$ ) of the model considered here; the interpolation found in [37] should correspond in our current language to a process in

which $q_{I}$ passes through a cut. (Moreover, the restriction to linear $m(z)$, etc., should be inessential to the behavior when $q_{I}$ is near a cut.) So this indicates that a process lowering $N_{i}$ by 1 by passing an eigenvalue through a cut can occur even when $N_{i}=2$. Subsequent work on $\mathcal{N}=2$ theories with matter [38 similarly indicates that such processes can occur for all $N_{i}>2$.

For $N_{i}=1$, however, we cannot expect to see a process in which yet another $q_{I}$ passes through a cut, reducing $N_{i}$ to zero. In such a process, the number of unbroken $U(1)$ factors in the low energy gauge group would change; this cannot occur continuously. It must be 
that the back reaction of a change in $m(z)$ on $\Sigma$ is crucial when a zero of $m(z)$ approaches a cut $A_{i}$ with $N_{i}=1$. This is not surprising, since the infrared behavior of $U(1)$ gauge theory with massless matter is completely different from that of $U(1)$ gauge theory without massless matter, so the effect of the zero of $m(z)$ on the cut will be large.

\section{The Theory With $W(\Phi)=0$}

As an application of the general results of the previous sections, we now study the special case of the theory with $W(\Phi)=0$. This theory has a moduli space of vacua $\mathcal{M}$ parametrized by $\Phi_{c l}$ modulo conjugation by a unitary matrix. Alternatively, $\mathcal{M}$ can be parametrized by the expectation values

$$
u_{k}=\frac{1}{k}\left\langle\operatorname{Tr} \Phi^{k}\right\rangle, \quad k=1, \ldots, N .
$$

Our goal is to compute the various observables as a function of $\Phi_{c l}$ or $u_{k}$.

We start by examining the structure of $\mathcal{M}$ in the classical theory. At a generic point in $\mathcal{M}$, the fundamental $U(N)$ gauge symmetry is broken to $U(1)^{N}$ and all the quarks are massive. At special points, there are singularities. If $k$ of the eigenvalues of $\Phi_{c l}$ are equal, there is in addition an unbroken $S U(k)$ symmetry. When an eigenvalues of $\Phi_{c l}$ coincides with one of the zeros of $\widehat{B}(z)$, say $z_{I}$, there is also a massless "electron."

In the quantum theory, we expect the $S U(k)$ singularities to be replaced by monopole points, but the points with massless electrons are IR free and are essentially unchanged. This semiclassical picture is identical to that in the $U(N) \mathcal{N}=2$ theory with $L$ flavors. Therefore, we expect the moduli space to be described by the hyperelliptic curve $y_{0}^{2}=$ $\operatorname{det}\left(z-\Phi_{c l}\right)^{2}-4 \Lambda^{2 N-N_{f}} \widehat{B}(z)$. We will soon see that this is indeed the case. It is therefore clear that if $L>2 N$ the metric on $\mathcal{M}$ is not positive definite at large $\Phi$ and the theory is not sensible. Note that this can happen even for $N_{f}<2 N$ where the gauge coupling

is asymptotically free. For arbitrary polynomials $m_{f}^{\widetilde{f}}$, the standard link between the beta function and the behavior of $\tau$ on the moduli space is not present.

As in [7], in order to study the theory at a generic point in $\mathcal{M}$, we deform it with a generic superpotential $W(\Phi)$ of degree $N+1$. We parametrize it as

$$
W^{\prime}(z)=g_{N} \prod_{i=1}^{N}\left(z-a_{i}\right)
$$


We study the theory for small $g_{N}$ and then set $g_{N}$ to zero. We consider the vacuum in which the $U(N)$ gauge symmetry is broken to $U(1)^{N}$, i.e. in which all $N_{i}=1$. This leads to

$$
\begin{aligned}
& y^{2}=W^{\prime}(z)^{2}+f(z)=g_{N}^{2} y_{0}^{2} \\
& y_{0}^{2}=P^{2}(z)-4 \Lambda^{2 N-N_{f}} \widehat{B}(z), \quad P(z)=\operatorname{det}\left(z-\Phi_{c l}\right)
\end{aligned}
$$

Equation (9.3) follows from (5.17) since $Q(z)$ and $H(z)$ are constants in this case.

For $L=\operatorname{deg}(\widehat{B}) \leq N-1$ this equation easily determines $W^{\prime}(z)=g_{N} P(z)$ and $f(z)=$ $-4 g_{N} \Lambda^{2 N-N_{f}} \widehat{B}(z)$. Starting with a tree level superpotential with parameters $a_{i}$ in (9.2), the vacuum of the system is at a point in $\mathcal{M}$ which is labeled by $\Phi_{c l}=\operatorname{diag}\left(a_{1}, a_{2}, \ldots, a_{N}\right)$. When we examine $T(z)$ below, we will see that for $k=1, \ldots, N$ in this case $u_{k}=\frac{1}{k} \operatorname{Tr} \Phi_{c l}^{k}$. For $N \leq L<2 N$ this identification is not so easy. The eigenvalues of $\Phi_{c l}$ are not the same as $a_{i}$ in (9.2) and $u_{k}=\frac{1}{k}\left\langle\operatorname{Tr} \Phi^{k}\right\rangle \neq \operatorname{Tr} \Phi_{c l}^{k}$.

It is clear that $R(z)$ is proportional to $g_{N}$ and therefore it vanishes when $g_{N} \rightarrow 0$. Similarly, $M(z)$ is proportional to $g_{N}$ and it vanishes in this limit. However,

$$
T(z)=\frac{\partial}{\partial z} \log \left(P(z)+y_{0}(z)\right)=\frac{P^{\prime}(z)}{y_{0}(z)}+\frac{\widehat{B}^{\prime}(z)}{2 \widehat{B}(z)}-\frac{P(z) \widehat{B}^{\prime}(z)}{2 y_{0}(z) \widehat{B}(z)}
$$

has a smooth nonzero limit as $g_{N} \rightarrow 0$, and therefore (9.4) is valid also when $W=0$.

As a check, from (9.3) we learn that $y_{0}\left(z_{I}\right)=P\left(z_{I}\right)$ (recall that we use the value in the first sheet), and since $P(z)$ is a polynomial,

$$
\sum_{I=1}^{L} \frac{y_{0}\left(z_{I}\right)}{2\left(z-z_{I}\right)}=\sum_{I=1}^{L} \frac{P\left(z_{I}\right)}{2\left(z-z_{I}\right)}=\left[\frac{P(z) \widehat{B}^{\prime}(z)}{2 \widehat{B}(z)}\right]_{-}
$$

Using this in $(3.7)$ equation $(3.10)$ becomes

$$
T(z)=\frac{\widehat{B}^{\prime}(z)}{2 \widehat{B}(z)}-\frac{P(z) \widehat{B}^{\prime}(z)}{2 y_{0}(z) \widehat{B}(z)}+\frac{\widetilde{c}(z)}{y_{0}(z)}
$$

with another polynomial $\widetilde{c}(z)$. It agrees with our general solution (9.4) for $\widetilde{c}(z)=P^{\prime}(z)$.

Now that we know $T(z)$, we can calculate

$$
u_{k}=\frac{1}{k}\left\langle\operatorname{Tr} \Phi^{k}\right\rangle=\frac{1}{2 \pi i k} \oint d z z^{k} T(z) .
$$

For $L<N$, we easily find that the gauge invariant coordinates on $\mathcal{M}$ are given by $u_{k}=$ $\frac{1}{k} \operatorname{Tr} \Phi_{c l}^{k}(k=1, \ldots, N)$. The observables $u_{k}$ with $k>N$ are not given by their classical 
values $\frac{1}{k} \operatorname{Tr} \Phi_{c l}^{k}$, but receive quantum corrections which are polynomials in the instanton factor $\Lambda^{2 N-N_{f}}$. These are quantum deformations of the classical relations in the chiral ring. This generalizes the result of [33] in the pure gauge theory to the case with matter.

For $N \leq L<2 N$, we find a more dramatic result. Even the coordinates on $\mathcal{M}$, which are the generators of the chiral ring, are not given by the classical expressions but receive instanton corrections $u_{k}=\frac{1}{k} \operatorname{Tr} \Phi_{c l}^{k}+\mathcal{O}\left(\Lambda^{2 N-N_{f}}\right)(k=1, \ldots, N)$.

As we said above, these results are the same as in the $\mathcal{N}=2$ theory with $L$ flavors. Equation (9.4) is thus an expression for $T(z)$ in the $\mathcal{N}=2$ theory with matter.

Now that we have explored the theory with $W(\Phi)=0$, we can turn on an arbitrary superpotential $W(\Phi)$. The effect of this superpotential can be analyzed as a small perturbation. From the structure of our equations is it clear that it cannot change the value of $T$ and that $R$ and $M$ are linear in $g_{n}$. The main effect of the superpotential is to choose vacua - choose $\Phi_{c l}$ in $\mathcal{M}$. Then various monopoles and electrons at that point condense and lift some of the photons in these vacua. But the curve $y_{0}^{2}=P(z)^{2}-4 \Lambda^{2 N-N_{f}} \widehat{B}(z)$ in these vacua is unchanged. The theory with nonzero $W$ inherits the curve from the theory with vanishing $W$.

\section{Acknowledgements}

It is a pleasure to thank M. Douglas and J. Maldacena for helpful discussions. This work was supported in part by DOE grant \#DE-FG02-90ER40542 and NSF grant PHY0070928 to IAS.

\section{Appendix A. Riemann Bilinear Relations.}

In this appendix we will give a brief review of the main tools from the theory of Riemann surfaces used in this work. Most of the results presented here are standard and are given for the reader's convenience. For this review we followed 443.

A Riemann surface of genus $g$ can be thought of as a $4 g$ polygon with some identifications of its edges. This representation is particularly useful to get relations among the integral of meromorphic one-forms along various cycles of the Riemann surface. These are known as Riemann bilinear relations. Although, this requires a particular choice of cycles and therefore breaking of modular invariance explicitly, the relations obtained this way can then be interpreted in any other basis of cycles by properly deforming the contours. This is the source of the integer ambiguity in all the formulas that refer to it in the main 
text of this paper. In sections 3 and 5 where the different cycles are explicit, the same choice has been made, which is consistent with the discussion in this appendix.

For our purposes it is enough to consider meromorphic one-forms with at most simple poles. Let $\mathcal{H}$ have simple poles at $T$ and $Q$ and $\mathcal{G}$ to have simple poles at $R$ and $S$. The Riemann surface $\Sigma$ of genus $g$ on which $\mathcal{H}$ is defined has puncture at $T$ and $Q$. In order to get a simply connected representation of it, we introduce cuts as shown in figure 4 , the new surface $\Sigma^{\prime}$ is simply connected and $\mathcal{H}$ can be written as $d f$ on it.

Let us compute $\int_{\partial \Sigma^{\prime}} f \mathcal{G}$, where $\partial \Sigma^{\prime}$ is the boundary of $\Sigma^{\prime}$ shown in figure 4 . We can evaluate this in two different ways. The first is to deform the contour to enclose the poles of $\mathcal{G}$ which we assume not to be located on $\partial \Sigma^{\prime}$. Using Cauchy's formula this leads to,

$$
\int_{\partial \Sigma^{\prime}} f \mathcal{G}=2 \pi i f(R) \operatorname{res}_{R} \mathcal{G}+2 \pi i f(S) \operatorname{res}_{S} \mathcal{G}
$$

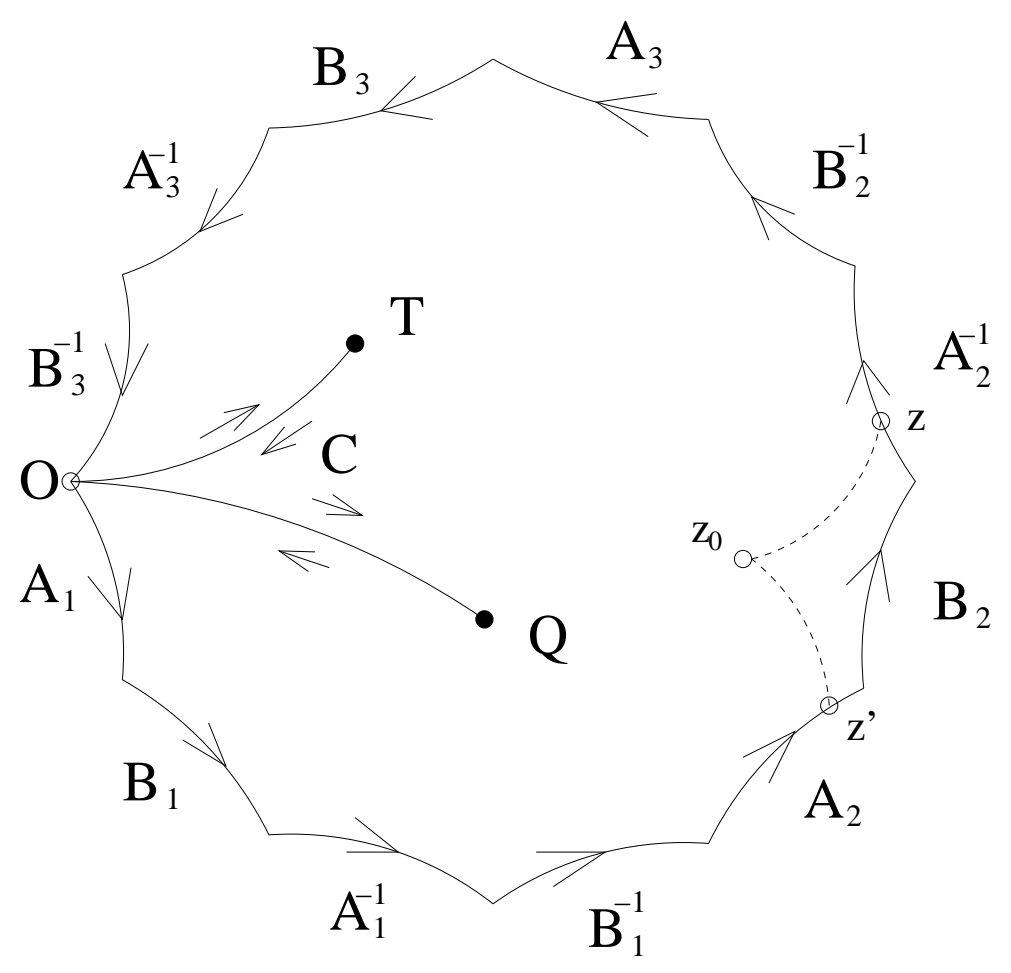

Fig. 4: Polygon $\Sigma^{\prime}$ representing a Riemann surface $\Sigma$ of genus $g=3$. Cuts from $O$ to $T$ and to $Q$ are introduced in order to make $\Sigma^{\prime}$ simply connected. The path $C$ runs from $O$ to $T$ and back around the cut $O T$ and from $O$ to $Q$ and back around the cut $O Q$. Also shown is the reference point $z_{0}$ in the definition of $f(z)$. The points $z$ and $z^{\prime}$ are identified. Dashed lines represent the contours used in integrals below. 
Since $\mathcal{G}$ does not have any more poles, $\operatorname{res}_{R} \mathcal{G}=-\operatorname{res}_{S} \mathcal{G}$. Using $\mathcal{H}=d f$ we can write (A.1) as,

$$
\int_{\partial \Sigma^{\prime}} f \mathcal{G}=2 \pi i\left(\operatorname{res}_{R} \mathcal{G} \int_{S}^{R} \mathcal{H}\right)
$$

The second way is to use the contour in figure 4. From there we conclude that,

$$
\int_{\partial \Sigma^{\prime}} f \mathcal{G}=\sum_{j=1}^{g}\left(\int_{A_{j}} f \mathcal{G}+\int_{B_{j}} f \mathcal{G}+\int_{A_{j}^{-1}} f \mathcal{G}+\int_{B_{j}^{-1}} f \mathcal{G}\right)+\int_{C} f \mathcal{G}
$$

Letting $f(z)=\int_{z_{0}}^{z} \mathcal{H}$ where $z_{0}$ is an arbitrary point in $\Sigma^{\prime}$ (see figure 4 ), we can compute,

$$
\int_{A_{j}} f \mathcal{G}+\int_{A_{j}^{-1}} f \mathcal{G}=\int_{A_{j}}\left(\int_{z_{0}}^{z} \mathcal{H}-\int_{z_{0}}^{z^{\prime}} \mathcal{H}\right) \mathcal{G}
$$

where $z$ and $z^{\prime}$ are points on $A_{j}$ and $A_{j}^{-1}$ respectively that are identified (see figure 4). The two integrals of $\mathcal{H}$ can be deformed to a single integral over the $B_{j}$ cycle to give,

$$
\int_{A_{j}} f \mathcal{G}+\int_{A_{j}^{-1}} f \mathcal{G}=-\int_{B_{j}} \mathcal{H} \int_{A_{j}} \mathcal{G}
$$

Likewise,

$$
\int_{B_{j}} f \mathcal{G}+\int_{B_{j}^{-1}} f \mathcal{G}=\int_{B_{j}} \mathcal{G} \int_{A_{j}} \mathcal{H}
$$

The last integral in (A.3) runs over the contour $C$ and can be computed by realizing that the value of $f$ on one side of the cut $O T$ differs from the value on the other side by $\oint_{T} \mathcal{H}$. Similarly, it differs by $\oint_{Q} \mathcal{H}$ between the two sides of the cut $O Q$. This implies that,

$$
\int_{C} f \mathcal{G}=\oint_{T} \mathcal{H} \int_{O}^{T} \mathcal{G}+\oint_{Q} \mathcal{H} \int_{O}^{Q} \mathcal{G}=\operatorname{res}_{T} \mathcal{H} \int_{Q}^{T} \mathcal{G}
$$

Combining (A.3), A.5), (A.6) and (A.7) we have,

$$
\int_{\partial \Sigma^{\prime}} f \mathcal{G}=\sum_{i=1}^{g}\left(\oint_{A_{i}} \mathcal{H} \oint_{B_{i}} \mathcal{G}-\oint_{A_{i}} \mathcal{G} \oint_{B_{i}} \mathcal{H}\right)+2 \pi i \operatorname{res}_{T} \mathcal{H} \int_{Q}^{T} \mathcal{G}
$$

Here we have also assumed that $\mathcal{H}$ does not have any more poles.

Comparing (A.2) and (A.8) we get the Riemann bilinear relation which all relations used in this work are special cases of,

$$
2 \pi i \operatorname{res}_{R} \mathcal{G} \int_{S}^{R} \mathcal{H}-2 \pi i \operatorname{res}_{T} \mathcal{H} \int_{Q}^{T} \mathcal{G}=\sum_{i=1}^{g}\left(\oint_{A_{i}} \mathcal{H} \oint_{B_{i}} \mathcal{G}-\oint_{A_{i}} \mathcal{G} \oint_{B_{i}} \mathcal{H}\right)
$$


Before illustrating the use of this formula, let us point out that the cycles used to define the edges of the 2g-polygon are not the same as the cycles in the double sheeted representation of the same genus g hyperelliptic Riemann surface. These two sets of cycles are in general related by an $S p(2 g, \mathbf{Z})$ transformation. We have not distinguished them since the right hand side of (A.9) is clearly $S p(2 g, \mathbf{Z})$ invariant. A more important distinction arises when the two sets of basis differ by the orientation of the resulting surfaces. In this case (A.9) can be used by reversing the orientation of the $B_{i}$ cycles. This will be important in the first application of (A.9) given next.

As a first illustration of the use of (A.9) let us derive (5.6). In this case we have $\mathcal{H}=\tau_{p_{1}, p_{2}}$ and $\mathcal{G}=\zeta_{k}$. Recall that $\tau_{p_{1}, p_{2}}$ has zero periods around the $A$ cycles and poles of order one with residue -1 and 1 at $p_{1}$ and $p_{2}$ respectively. On the other hand $\zeta_{k}$ is a holomorphic one form with $\oint_{A_{i}} \zeta_{k}=\delta_{i k}$. The choice of orientation in figure 2 is the opposite to that of figure 4 . Therefore, as discussed before, the sign of integrals over $B$ cycles should be reversed. Combining this in (A.9) we get (5.6),

$$
\frac{1}{2 \pi i} \oint_{B_{k}} \tau_{p_{1}, p_{2}}=\int_{p_{2}}^{p_{1}} \zeta_{k} .
$$

As a second illustration, let us derive (5.11). Take $\mathcal{H}=\tau_{T, Q}$ and $\mathcal{G}=\tau_{R, S}$. Recalling that these differentials have zero periods around the $A$ cycles, the right hand side of (A.9) is zero. This leads to,

$$
\operatorname{res}_{R} \tau_{R, S} \int_{S}^{R} \tau_{T, Q}=\operatorname{res}_{T} \tau_{T, Q} \int_{Q}^{T} \tau_{R, S} .
$$

Using that $\operatorname{res}_{R} \tau_{R, S}=-1$ and $\operatorname{res}_{T} \tau_{T, Q}=-1$ we get (5.11).

The derivation of (A.9) and therefore of (5.11) assumes that all points $R, S, T, Q$ are distinct. In section 3 we need a degenerate version of this, i.e., the case when $T=R$. The integral in (A.11) are divergent in this case and need a regulator. Without loss of generality we can choose local coordinates vanishing at $T$. Let us then take $R$ at $z=\epsilon$. For $\epsilon \neq 0$ we can use (A.11),

$$
\int_{S}^{\epsilon} \tau_{0, Q}=\int_{Q}^{0} \tau_{\epsilon, S} .
$$

We need to express the integral on the right hand side in terms of $\tau_{0, S}$ and a regularized contour. The two integrals are not the same but are related by exchanging the end of the contour with the location of the pole. This operation introduces the integral around half 
a contour around the pole and therefore gives a half. Let us check this explicitly. We need to compute,

$$
\int_{Q}^{0} \tau_{\epsilon, S}-\int_{Q}^{\epsilon} \tau_{0, S}
$$

In the local coordinates we chose, $\tau_{\epsilon, S}=-\frac{1}{z-\epsilon} d z+$ regular and $\tau_{0, S}=-\frac{1}{z} d z+$ regular. The regular terms will not contribute to (A.13) in the limit $\epsilon \rightarrow 0$, and therefore will not be considered. Explicit evaluation of (A.13) gives $-\log (-\epsilon)+\log \epsilon+\mathcal{O}(\epsilon)=-\pi i+\mathcal{O}(\epsilon)$. Therefore,

$$
\int_{Q}^{0} \tau_{\epsilon, S}=\int_{Q}^{\epsilon} \tau_{0, S}-\pi i+\mathcal{O}(\epsilon)
$$

Using this in (A.12) we get the general form of (5.13),

$$
\int_{S}^{\epsilon} \tau_{0, Q}=\int_{Q}^{\epsilon} \tau_{0, S}-\pi i+\mathcal{O}(\epsilon)
$$

\section{Appendix B. Sphere Contribution To Effective Superpotential}

In this appendix we will carry out the computation of $\frac{\partial \mathcal{F}_{0}}{\partial S_{i}}$ from the saddle point approximation result (6.9). The answer is used to compute the sphere contribution to the effective superpotential $W_{\text {eff }}$ of the field theory (7.1). We will use standard matrix model techniques to calculate $\mathcal{F}_{0}$ (for a review see e.g. [42]), and will express the answer in terms of contour integrals. Similar computations can be found elsewhere (e.g. in [19]), but we will keep terms that were not important for the analysis there, and were not explicitly shown. We will also manipulate only finite quantities making all dependence on the cutoff $\Lambda_{0}$ explicit.

Recall that our normalization for the eigenvalue density is $\int d \lambda \rho(\lambda)=S$. Therefore, the filling fractions are

$$
S_{j}=\int_{a_{j}^{-}}^{a_{j}^{+}} d \lambda \rho(\lambda)
$$

Consider (6.9) modified to include chemical potentials $\mu_{i}$.

$$
\mathcal{F}_{0}\left(\mu_{i}, \rho\right)=\int d \lambda \rho(\lambda) W(\lambda)-\iint d \lambda d \lambda^{\prime} \rho(\lambda) \rho\left(\lambda^{\prime}\right) \log \left|\frac{\lambda-\lambda^{\prime}}{\Lambda}\right|-\sum_{i=1}^{n} \mu_{i} \int_{a_{i}^{-}}^{a_{i}^{+}} d \lambda \rho(\lambda)
$$

This is a functional of $\rho(\lambda)$ and a function of $\mu_{i}$ 's. $S_{i}$ 's will be introduced via a Legendre transform. 
The variation of (B.2) with respect to $\rho(\lambda)$ with $\lambda$ a point in the $i$-th cut is

$$
\frac{\delta \mathcal{F}_{0}}{\delta \rho(\lambda)}=W(\lambda)-2 \int d \lambda^{\prime} \rho\left(\lambda^{\prime}\right) \log \left|\frac{\lambda-\lambda^{\prime}}{\Lambda}\right|-\mu_{i}=0
$$

This is the equation of motion of $\rho(\lambda)$. It follows from it that

$$
W^{\prime}(\lambda)-2 \mathcal{P} \int d \lambda^{\prime} \frac{\rho\left(\lambda^{\prime}\right)}{\lambda-\lambda^{\prime}}=0
$$

where $\mathcal{P}$ denotes the principal part.

Although we do not need it here, we add for completeness a derivation of the loop equation. By multiplying (B.4) by $\frac{\rho(\lambda)}{z-\lambda}$ and integrating over $\lambda$ we derive

$$
\begin{aligned}
\int d \lambda \frac{\rho(\lambda) W^{\prime}(\lambda)}{z-\lambda} & =2 \mathcal{P} \int d \lambda d \lambda^{\prime} \frac{\rho(\lambda) \rho\left(\lambda^{\prime}\right)}{(z-\lambda)\left(\lambda-\lambda^{\prime}\right)} \\
& =\mathcal{P} \int d \lambda d \lambda^{\prime} \frac{\rho(\lambda) \rho\left(\lambda^{\prime}\right)}{\lambda-\lambda^{\prime}}\left(\frac{1}{z-\lambda}-\frac{1}{z-\lambda^{\prime}}\right) \\
& =\int d \lambda d \lambda^{\prime} \frac{\rho(\lambda) \rho\left(\lambda^{\prime}\right)}{(z-\lambda)\left(z-\lambda^{\prime}\right)} \\
& =\left(\int d \lambda \frac{\rho(\lambda)}{z-\lambda}\right)^{2}
\end{aligned}
$$

Since $\rho(\lambda)=-\frac{1}{2 \pi i} \operatorname{disc} R(\lambda), \int d \lambda \rho(\lambda) F(\lambda)=\sum_{i} \frac{1}{2 \pi i} \oint_{A_{i}} d w R(w) F(w)$ for any function $F(w)$ which is analytic on the cuts $A_{i}$. A special case of this is $\int d \lambda \frac{\rho(\lambda)}{z-\lambda}=$ $\sum_{i} \frac{1}{2 \pi i} \oint_{A_{i}} d w \frac{R(w)}{z-w}=-\frac{1}{2 \pi i} \oint_{z} d w \frac{R(w)}{z-w}=R(z)$. Using $\int d \lambda \frac{\rho(\lambda) W^{\prime}(\lambda)}{z-\lambda}=\left[W^{\prime}(z) \int d \lambda \frac{\rho(\lambda)}{z-\lambda}\right]_{-}=$ $\left[W^{\prime}(z) R(z)\right]_{-}$, we derive from (B.5) the loop equation $\left[W^{\prime}(z) R(z)\right]_{-}=R(z)^{2}$.

From the equation of motion (B.3) we solve for $\langle\rho(\lambda)\rangle$ as a function of $\mu_{1}, \ldots, \mu_{n}$. This leads to the free energy with fixed chemical potentials

$$
\mathcal{F}_{0}\left(\mu_{1}, \ldots, \mu_{n}\right)=\mathcal{F}_{0}\left(\mu_{1}, \ldots, \mu_{n},\langle\rho(\lambda)\rangle\right) .
$$

Under a Legendre transformation

$$
\mathcal{F}_{0}\left(S_{1}, \ldots, S_{n}\right)=\mathcal{F}_{0}\left(\mu_{1}, \ldots, \mu_{n}\right)+\sum_{i=1}^{n} \mu_{i} S_{i}
$$

where

$$
\frac{\partial \mathcal{F}_{0}\left(\mu_{1}, \ldots, \mu_{n}\right)}{\partial \mu_{i}}+S_{i}=0
$$


is used to write $\mu_{i}=\mu_{i}\left(S_{1}, \ldots, S_{n}\right)$. The Legendre transform (equation of motion of $S_{i}$ ) determines

$$
\frac{\partial \mathcal{F}_{0}\left(S_{1}, \ldots, S_{n}\right)}{\partial S_{i}}=\mu_{i}
$$

Using (B.3) we find

$$
\frac{\partial \mathcal{F}_{0}\left(S_{1}, \ldots, S_{n}\right)}{\partial S_{i}}=W(\lambda)-2 \int d \lambda^{\prime} \rho\left(\lambda^{\prime}\right) \log \left|\frac{\lambda-\lambda^{\prime}}{\Lambda}\right| .
$$

Recall that $\lambda$ is a point in the $i$-th cut. However, the combination appearing in (B.10) is independent of $\lambda$ (see (B.4) ). Therefore, a convenient $\lambda$ can be chosen to evaluate (B.10). Take $\lambda=a_{i}^{+}$, i.e. one of the ends of the $i$-th cut. The integral over the cuts of $\rho\left(\lambda^{\prime}\right)$ can be replaced by a sum of integrals around the $A_{j}$ cycles of $R(z)$ as follows

$$
\frac{\partial \mathcal{F}_{0}\left(S_{1}, \ldots, S_{n}\right)}{\partial S_{i}}=W\left(a_{i}^{+}\right)-2 \sum_{j=1}^{n} \frac{1}{2 \pi i} \oint_{A_{j}} d z R(z) \log \left(\frac{z-a_{i}^{+}}{\Lambda}\right) .
$$

Note that the integrand is not an analytic function on the cut $A_{i}$ but the logarithmic singularity does not lead to a divergence there. The continuation of the function $\log \mid a_{i}^{+}-$ $\lambda^{\prime} \mid$ into a complex function might introduce more terms which are linear combinations of $2 \pi i S_{j}$ with integer coefficients. These terms are irrelevant for our computation since they correspond to a choice of the winding number of the regularized contours $\widehat{B}_{j}^{r}$ around the $A_{k}$ cycles as will be clear from the final expression.

We now deform the contours in (B.11) to a contour that starts at $\Lambda_{0}$ winds around on a large circle of radius $\left|\Lambda_{0}\right|$, moves in along the cut of the logarithm to $a_{i}^{+}$and back to $\Lambda_{0}$ along the other side of this cut. Using $R(z)=\frac{S}{z}+\mathcal{O}\left(1 / z^{2}\right)$, this leads to

$$
\begin{aligned}
\frac{\partial \mathcal{F}_{0}\left(S_{1}, \ldots, S_{n}\right)}{\partial S_{i}} & =W\left(a_{i}^{+}\right)+2 \int_{a_{i}^{+}}^{\Lambda_{0}} R(z) d z-2 S \log \left(-\frac{\Lambda_{0}}{\Lambda}\right)+\mathcal{O}\left(1 / \Lambda_{0}\right) \\
& =W\left(\Lambda_{0}\right)-\int_{a_{i}^{+}}^{\Lambda_{0}} y d z-2 S \log \left(-\frac{\Lambda_{0}}{\Lambda}\right)+\mathcal{O}\left(1 / \Lambda_{0}\right) .
\end{aligned}
$$

where we used $2 R(z)=W^{\prime}(z)-y(z)$. Since $y(z)$ vanishes at $a_{i}^{+}$, we can write it as

$$
\frac{\partial \mathcal{F}_{0}}{\partial S_{i}}=-\frac{1}{2} \int_{\widehat{B}_{i}^{r}} y(z) d z+W\left(\Lambda_{0}\right)-2 S \log \left(-\frac{\Lambda_{0}}{\Lambda}\right)+\mathcal{O}\left(1 / \Lambda_{0}\right) .
$$

Now that the answer is written as a contour integral, the contour can be moved through the cut $A_{i}$ and does not have to pass through $a_{i}^{+}$. 
Finally, we can write the sphere contribution to the effective superpotential from (7.1),

$$
\left.W_{\text {eff }}\right|_{\text {sphere }}=-\frac{1}{2} \sum_{i=1}^{n} N_{i} \int_{\widehat{B}_{i}^{r}} y(z) d z+2 \pi i \sum_{i=1}^{n-1} b_{i} S_{i}+N W\left(\Lambda_{0}\right)-2 N S \log \left(-\frac{\Lambda_{0}}{\Lambda}\right) .
$$

up to terms of order $1 / \Lambda_{0}$.

Before concluding this appendix let us note that the free energy $\mathcal{F}_{0}$ is a homogeneous function of degree 2 in the combination of $S_{1}, \ldots, S_{n}$ and $g_{0}, \ldots, g_{n}$. In order to see this, consider the definition,

$$
\exp \left(-\frac{\widehat{N}^{2}}{S^{2}} \mathcal{F}_{0}+\ldots\right)=\frac{1}{\operatorname{vol} U(\widehat{N})} \int \frac{d \widehat{\Phi}}{\Lambda^{N^{2}}} \exp \left(-\frac{\widehat{N}}{S} \operatorname{Tr} W(\widehat{\Phi})\right) .
$$

where the ellipses represent higher order terms in the $1 / \widehat{N}$ expansion.

Recalling that $W(z)=\sum_{k=0}^{n} \frac{g_{k}}{k+1} z^{k+1}$, it is simple to see that $\mathcal{F}_{0}$ can only depend on the couplings through the combination $g_{k} / S$. In addition, $\mathcal{F}_{0}$ can only depend on $S_{i}$ 's through the filling fractions $S_{i} / S$. Finally, note that from the exponential in (B.15), the free energy has to be of the form,

$$
\mathcal{F}_{0}=S^{2} f\left(\frac{g_{0}}{S}, \ldots, \frac{g_{n}}{S}, \frac{S_{1}}{S}, \ldots, \frac{S_{n}}{S}\right) .
$$

This proves the statement that $\mathcal{F}_{0}$ is homogeneous of degree 2 in $g_{i}$ 's and $S_{i}$ 's; i.e.

$$
2 \mathcal{F}_{0}=\sum_{k=0}^{n} g_{k} \frac{\partial \mathcal{F}_{0}}{\partial g_{k}}+\sum_{i=1}^{n} S_{i} \frac{\partial \mathcal{F}_{0}}{\partial S_{i}}
$$

\section{Appendix C. Strong Coupling Analysis}

Once $T(z)$ has been found for the theory without superpotential, we can repeat the strong coupling analysis of [34] for the case when the deformation is by $W(\Phi)$ of degree $N+1$. This will be a consistency check of the previous results since it leads to the identification of the curves as in (9.3) and it is carried out in the opposite limit, i.e. in the strong coupling regime. The main reason this computation is nontrivial is that in the quantum theory $W(\Phi)$ is not given by $W\left(\Phi_{c l}\right)$ but we have to be careful about the instanton corrections.

The superpotential for $\Phi$ is introduced as a small deformation of the theory. This deformation leads to an effective superpotential for the vev's of $\frac{1}{k} \operatorname{Tr} \Phi^{k}$ denoted by $U_{k}$. 
These vev's are computed from the generating function

$$
\left\langle\operatorname{Tr} \frac{1}{z-\Phi}\right\rangle=\frac{N}{z}+\sum_{l=1}^{\infty} \frac{l U_{l}}{z^{l+1}}
$$

From $(5.20)$ we also have

$$
\left\langle\operatorname{Tr} \frac{1}{z-\Phi}\right\rangle=\frac{\partial}{\partial z} \log \left(P(z)+\sqrt{P^{2}(z)-4 \Lambda^{2 N-N_{f}} \widehat{B}(z)}\right)
$$

Using (C.1) and (C.2) we get

$$
P(z)=z^{N} \exp \left(-\sum_{i=1}^{\infty} \frac{U_{i}}{z^{i}}\right)+\Lambda^{2 N-N_{f}} \frac{\widehat{B}(z)}{z^{N}} \exp \left(\sum_{i=1}^{\infty} \frac{U_{i}}{z^{i}}\right)
$$

Given that $P(z)$ is a polynomial of degree $N$, (C.2) can be thought of as determining the coefficients of $P(z)$ and $U_{k}$ for $k \geq N+1$ in terms of $U_{l}$ for $l=1, \ldots, N$. It is important to note that $P(z)$ is not equal to $\langle\operatorname{det}(z-\Phi)\rangle$. From (C.3) the relation can be seen to be

$$
P(z)=\langle\operatorname{det}(z-\Phi)\rangle+\left[\Lambda^{2 N-N_{f}} \frac{\widehat{B}(z)}{z^{N}} \exp \left(\sum_{i=1}^{\infty} \frac{U_{i}}{z^{i}}\right)\right]_{+}
$$

In order to use the deformation by the superpotential $W(\Phi)$ to fix a point in the space of $U_{k}$ 's for $k=1, \ldots, N$, we have to take the degree of $W$ to be $N+1$. This implies that $U_{N+1}$ has to be written in terms of the lower traces. We will consider all $U_{k}$ 's to be independent and impose the relation from (C.3) as a constraint.

The deformation by the superpotential $W(\Phi)$ gives an effective superpotential for $U_{k}$ 's of the form

$$
W=\sum_{r=0}^{N} g_{r} U_{r+1}+R_{0} \oint\left(z^{N} \exp \left(-\sum_{i=1}^{\infty} \frac{U_{i}}{z^{i}}\right)+\Lambda^{2 N-N_{f}} \frac{\widehat{B}(z)}{z^{N}} \exp \left(\sum_{i=1}^{\infty} \frac{U_{i}}{z^{i}}\right)\right) d z
$$

where $R_{0}$ is a Lagrange multiplier imposing the constraint that enforces $U_{N+1}$ to be a function of the lower $U_{l}$ 's.

Taking variations with respect to $U_{r+1}$, we get

$$
g_{r}=R_{0} \oint\left(z^{N-r-1} \exp \left(-\sum_{i=1}^{\infty} \frac{U_{i}}{z^{i}}\right)-\Lambda^{2 N-N_{f}} \frac{\widehat{B}(z)}{z^{N+r+1}} \exp \left(\sum_{i=1}^{\infty} \frac{U_{i}}{z^{i}}\right)\right) d z
$$


Using this we can compute $W^{\prime}(x)=\sum_{r=0}^{N} g_{r} x^{r}$ to be

$$
W^{\prime}(x)=\oint\left(z^{N} \exp \left(-\sum_{i=1}^{\infty} \frac{U_{i}}{z^{i}}\right)-\Lambda^{2 N-N_{f}} \frac{\widehat{B}(z)}{z^{N}} \exp \left(\sum_{i=1}^{\infty} \frac{U_{i}}{z^{i}}\right)\right) \frac{d z}{(z-x)}
$$

where we have assumed that $L \leq 2 N$ in order to extend the sums over $r$ from $N$ to infinity and perform the sum to get $(z-x)^{-1}$. Here $z$ is assumed to be inside the contour of integration.

On the constraint surface,

$$
z^{N} \exp \left(-\sum_{i=1}^{\infty} \frac{U_{i}}{z^{i}}\right)=\frac{1}{2}\left(P(z)+\sqrt{P^{2}(z)-4 \Lambda^{2 N-N_{f}} \widehat{B}(z)}\right) .
$$

Using this in (C.7), we get

$$
W^{\prime}(z)=R_{0} \oint \frac{\sqrt{P^{2}(z)-4 \Lambda^{2 N-N_{f}} \widehat{B}(z)}}{(z-x)} d z
$$

Letting $g_{N}=1$, this equation implies that

$$
y^{2}=P^{2}(z)-4 \Lambda^{2 N-N_{f}} \widehat{B}(z)=W^{\prime}(z)^{2}+\mathcal{O}\left(x^{N-1}\right) .
$$

This is consistent with the result found in (5.17) and (9.3), i.e.

$$
y^{2}=P^{2}(z)-4 \Lambda^{2 N-N_{f}} \widehat{B}(z)=W^{\prime}(z)^{2}+f(z) .
$$




\section{References}

[1] K. A. Intriligator and N. Seiberg, "Lectures on supersymmetric gauge theories and electric-magnetic duality," Nucl. Phys. Proc. Suppl. 45BC, 1 (1996) arXiv:hepth/9509066].

[2] R. Dijkgraaf and C. Vafa, "A perturbative window into non-perturbative physics," arXiv:hep-th/0208048.

[3] M. Bershadsky, S. Cecotti, H. Ooguri and C. Vafa, "Kodaira-Spencer theory of gravity and exact results for quantum string amplitudes," Commun. Math. Phys. 165, 311 (1994) arXiv:hep-th/9309140.

[4] R. Gopakumar and C. Vafa, "On the gauge theory/geometry correspondence," Adv. Theor. Math. Phys. 3, 1415 (1999) arXiv:hep-th/9811131.

[5] C. Vafa, "Superstrings and topological strings at large N," J. Math. Phys. 42, 2798 (2001) arXiv:hep-th/0008142.

[6] F. Cachazo, K. A. Intriligator and C. Vafa, "A large N duality via a geometric transition," Nucl. Phys. B 603, 3 (2001) arXiv:hep-th/0103067.

[7] F. Cachazo and C. Vafa, "N $=1$ and $\mathrm{N}=2$ geometry from fluxes," arXiv:hepth/0206017.

[8] R. Dijkgraaf and C. Vafa, "Matrix models, topological strings, and supersymmetric gauge theories," arXiv:hep-th/0206255.

[9] R. Dijkgraaf and C. Vafa, "On geometry and matrix models," arXiv:hep-th/0207106.

[10] T. J. Hollowood and T. Kingaby, "The phase structure of mass-deformed SU(2) x $\mathrm{SU}(2)$ quiver theory," arXiv:hep-th/0210096.

[11] D. Berenstein, "Quantum moduli spaces from matrix models," arXiv:hep-th/0210183.

[12] R. Argurio, V. L. Campos, G. Ferretti and R. Heise, "Exact superpotentials for theories with flavors via a matrix integral," arXiv:hep-th/0210291.

[13] J. McGreevy, "Adding flavor to Dijkgraaf-Vafa," arXiv:hep-th/0211009.

[14] H. Suzuki, "Perturbative derivation of exact superpotential for meson fields from matrix theories with one flavour," arXiv:hep-th/0211052.

[15] I. Bena and R. Roiban, "Exact superpotentials in $\mathrm{N}=1$ theories with flavor and their matrix model formulation," arXiv:hep-th/0211075.

[16] Y. Demasure and R. A. Janik, "Effective matter superpotentials from Wishart random matrices," arXiv:hep-th/0211082.

[17] Y. Tachikawa, "Derivation of the Konishi anomaly relation from Dijkgraaf-Vafa with (bi-)fundamental matters," arXiv:hep-th/0211189.

[18] R. Argurio, V. L. Campos, G. Ferretti and R. Heise, "Baryonic corrections to superpotentials from perturbation theory," arXiv:hep-th/0211249. 
[19] S. G. Naculich, H. J. Schnitzer and N. Wyllard, "Matrix model approach to the N $=2 \mathrm{U}(\mathrm{N})$ gauge theory with matter in the fundamental representation," arXiv:hepth/0211254.

[20] I. Bena, R. Roiban and R. Tatar, "Baryons, boundaries and matrix models," arXiv:hep-th/0211271.

[21] B. Feng, "Seiberg duality in matrix model," arXiv:hep-th/0211202.

[22] B. Feng and Y. H. He, "Seiberg duality in matrix models. II," arXiv:hep-th/0211234.

[23] Y. Ookouchi, "N = 1 gauge theory with flavor from fluxes," arXiv:hep-th/0211287.

[24] K. Ohta, "Exact mesonic vacua from matrix models," arXiv:hep-th/0212025.

[25] I. Bena, S. de Haro and R. Roiban, "Generalized Yukawa couplings and matrix models," arXiv:hep-th/0212083.

[26] C. Hofman, "Super Yang-Mills with flavors from large N(f) matrix models," arXiv:hepth/0212095.

[27] N. Seiberg, "Adding fundamental matter to 'Chiral rings and anomalies in supersymmetric gauge theory"," arXiv:hep-th/0212225.

[28] B. Feng, "Note on matrix model with massless flavors," arXiv:hep-th/0212274.

[29] R. Roiban, R. Tatar and J. Walcher, "Massless flavor in geometry and matrix models," arXiv:hep-th/0301217.

[30] V. Balasubramanian, B. Feng, M. x. Huang and A. Naqvi, "Phases of N=1 Supersymmetric Gauge Theories with Flavors," arXiv:hep-th/0303065.

[31] I. Bena, H. Murayama, R. Roiban and R. Tatar, "Matrix Model Description of Baryonic Deformations," arXiv:hep-th/0303115.

[32] B. Feng, "Note on Seiberg Duality in Matrix Model," arXiv:hep-th/0303144.

[33] F. Cachazo, M. R. Douglas, N. Seiberg and E. Witten, "Chiral rings and anomalies in supersymmetric gauge theory," arXiv:hep-th/0211170.

[34] F. Cachazo, N. Seiberg and E. Witten, "Phases of $\mathrm{N}=1$ supersymmetric gauge theories and matrices," arXiv:hep-th/0301006.

[35] E. H. Fradkin and S. H. Shenker, "Phase Diagrams Of Lattice Gauge Theories With Higgs Fields," Phys. Rev. D 19, 3682 (1979).

[36] T. Banks and E. Rabinovici, "Finite Temperature Behavior Of The Lattice Abelian Higgs Model," Nucl. Phys. B 160, 349 (1979).

[37] N. Seiberg and E. Witten, "Monopoles, duality and chiral symmetry breaking in N=2 supersymmetric QCD," Nucl. Phys. B 431, 484 (1994) arXiv:hep-th/9408099.

[38] A. Hanany and Y. Oz, "On the quantum moduli space of vacua of $\mathrm{N}=2$ supersymmetric SU(N(c)) gauge theories," Nucl. Phys. B 452, 283 (1995) arXiv:hep-th/9505075. P. C. Argyres, M. R. Plesser and A. D. Shapere, "The Coulomb phase of $\mathrm{N}=2$ supersymmetric QCD," Phys. Rev. Lett. 75, 1699 (1995) arXiv:hep-th/9505100. J. A. Minahan and D. Nemeschansky, "Hyperelliptic curves for supersymmetric YangMills," Nucl. Phys. B 464, 3 (1996) arXiv:hep-th/9507032. I. M. Krichever and 
D. H. Phong, "On the integrable geometry of soliton equations and $\mathrm{N}=2$ supersymmetric gauge theories," J. Diff. Geom. 45, 349 (1997) arXiv:hep-th/9604199. E. D'Hoker, I. M. Krichever and D. H. Phong, "The effective prepotential of $\mathrm{N}=2$ supersymmetric SU(N(c)) gauge theories," Nucl. Phys. B 489, 179 (1997) arXiv:hepth/9609041.

[39] A. Kapustin, "The Coulomb branch of $\mathrm{N}=1$ supersymmetric gauge theory with adjoint and fundamental matter," Phys. Lett. B 398, 104 (1997) arXiv:hep-th/9611049.

[40] I.G. Macdonald, "The volume of a compact Lie group," Invent. Math. 56, 93 (1980).

[41] H. Ooguri and C. Vafa, "Worldsheet derivation of a large N duality," Nucl. Phys. B 641, 3 (2002) arXiv:hep-th/0205297.

[42] P. Di Francesco, P. Ginsparg and J. Zinn-Justin, "2-D Gravity and random matrices," Phys. Rept. 254, 1 (1995) arXiv:hep-th/9306153.

[43] H.M. Farkas and I. Kra. Riemann Surfaces, 2nd ed., Springer-Verlag, 1992. 